\title{
Spatial and molecular profiling reveals an immunosuppressive mononuclear phagocyte network in Classic Hodgkin lymphoma
}

\section{Benjamin Stewart}

University of Cambridge

Martin Fergie

University of Manchester

Matthew Young

Wellcome Trust Sanger Institute https://orcid.org/0000-0003-0937-5290

Claire Jones

Newcastle upon Tyne NHS Foundation Trust

\section{Ashwin Sachdeva}

University of Manchester

Alex Blain

Newcastle University

Chris Bacon

Newcastle University

Vikki Rand

Teeside University

John Ferdinand

University of Cambridge

Kylie James

Garvan Institute of Medical Research

Krishnaa Mahbubani

University of Cambridge

Liz Hook

University of Cambridge

Nicolaas Jonas

University of Cambridge

Nicholas Coleman

University of Cambridge

Kourosh Saeb-Parsy

Cambridge University https://orcid.org/0000-0002-0633-3696

Matthew Collin 
University of Newcastle https://orcid.org/0000-0001-6585-9586

\section{Menna Clatworthy}

University of Cambridge

\section{Sam Behjati}

Wellcome Sanger Institute https://orcid.org/0000-0002-6600-7665

Christopher Carey ( $\nabla$ christopher.carey@newcastle.ac.uk)

Newcastle University https://orcid.org/0000-0002-4293-3920

\section{Short Report}

Keywords: Lymphoid neoplasia, Hodgkin lymphoma, Hodgkin's disease, Classic Hodgkin lymphoma, tumor microenvironment, microenvironment, dendritic cells, mononuclear phagocytes, classical monocytes, macrophages, Hodgkin cells, immunobiology, immunotherapy, Transcriptomics, proteomics

Posted Date: August 26th, 2022

DOI: https://doi.org/10.21203/rs.3.rs-1233380/v3

License: (c) (1) This work is licensed under a Creative Commons Attribution 4.0 International License. Read Full License 


\section{Abstract}

Classic Hodgkin lymphoma ( $\mathrm{cHL}$ ) has a rich immune infiltrate, which is an intrinsic component of the neoplastic process. Malignant Hodgkin Reed-Sternberg cells (HRSC) create an immunosuppressive microenvironment by the expression of regulatory molecules, preventing T-cell activation. It has also been demonstrated that mononuclear phagocytes (MNP) in the vicinity of HRSC express similar regulatory mechanisms in parallel, and their presence in tissue is associated with inferior patient outcomes. MNP in $\mathrm{cHL}$ have hitherto been identified with a small number of canonical markers and are usually described as 'tumor-associated macrophages'.

The organization of MNP networks and interactions with HRSC remains unexplored at high resolution. Here, we defined the global immune cell composition of $\mathrm{cHL}$ and non-lymphoma lymph nodes, integrating data across single-cell RNA sequencing, spatial transcriptomics and multiplexed immunofluorescence. We observed that MNP comprise multiple subsets of monocytes, macrophages and dendritic cells (DC). Classical monocytes, macrophages and conventional DC2 were enriched in the vicinity of HRSC, but plasmacytoid DC and activated DC were excluded.

Unexpectedly, CDC and monocytes expressed immunoregulatory checkpoints PD-L1, TIM-3, and the tryptophan-catabolizing protein IDO, at the same level as macrophages. Expression of these molecules increased with age. We also found that classical monocytes are important signaling hubs, potentially controlling the retention of CDC2 and ThExh via CCR1-, CCR4-, CCR5-, and CXCR3-dependent signaling. Enrichment of the $\mathrm{CDC2}$-monocyte-macrophage network in diagnostic biopsies is associated with early treatment failure. These results reveal unanticipated complexity and spatial polarization within the MNP compartment, further demonstrating their potential roles in immune evasion by $\mathrm{cHL}$.

\section{Key Points}

- Tumor-associated MNP are diverse and spatially polarized: CDC2 colocalize with malignant HRSC; plasmacytoid and activated DC are excluded.

- DC, monocytes, and macrophages exist in an inflammatory niche in close proximity to HRSC, and all express inhibitory molecules.

\section{Introduction}

Classic Hodgkin lymphoma ( $\mathrm{cHL}$ ) accounts for $12-15 \%$ of lymphoma diagnoses and occurs most frequently in adolescents and young adults, with another peak of incidence in older adults. Multi-agent cytotoxic chemotherapy (including selective use of radiotherapy) is curative in $80-95 \%$ of patients; in adults the potential for cure is largely determined by the disease stage ${ }^{1-3}$. However, for patients with 
relapsed or refractory disease, the prospect of cure or long-term disease control is significantly diminished.

A cHL tumor is defined by CD30+ Hodgkin Reed-Sternberg Cells (HRSC), comprising $1-5 \%$ of cells, and derived from germinal center (GC) B cells ${ }^{4}$. HRSC survive within a tumor microenvironment (TME) containing abundant T-cells and a network of mononuclear phagocytes (MNP) ${ }^{4}$. HRSC establish an immunosuppressive niche through multiple mechanisms, including downregulation of major histocompatibility complex (MHC) proteins and enhanced expression of programmed cell death ligand-1 (PD-L1), resulting from 9p23-p24 copy number gain or amplification ${ }^{5,6}$. PD-L1 is also abundantly expressed by myeloid cells surrounding HRSC, leading to the notion that MNP in $\mathrm{CHL}$ are largely tumorassociated macrophages (TAM) $)^{7,8}$.

Recent cross-tissue surveys of MNP diversity, using single cell approaches, have characterized multiple states of monocytes, macrophages, and dendritic cells, identifying significant differences in functional properties and roles in pathology ${ }^{8}$. Although MNP are frequently observed in $\mathrm{cHL}$, details of their functional specialization are not known.

Here, we use complementary high-resolution techniques to map MNP diversity in the microenvironment of $\mathrm{cHL}$. We find that tumor-associated MNP comprise multiple cell-types, occupy distinct and prognostically relevant niches, and find that MNPs adjacent to HRSC exhibit immunosuppressive phenotypes. These results reveal the complexity of MNP networks in $\mathrm{CHL}$, and critical intercellular communication pathways that may be therapeutically tractable.

\section{Methods}

Tissue samples for scRNAseq

Healthy lymph nodes, unaffected by neoplastic disease, were acquired from donation after circulatory death (DCD) adult donors at the time of organ retrieval for transplantation by the Cambridge Biorepository for Translational Medicine (CBTM) with ethical approval (15/EE/0152, East of EnglandCambridge South Research Ethics Committee) and consent from donor families (Table 1). Lymph node samples were collected from inguinal, mesenteric, and thoracic regions at the end of organ donation, within 1-2 hours of cessation of circulation under cold ischaemic conditions. Lymph node specimens were transported in ice-cold $0.9 \%$ saline. Tissue was processed as previously described ${ }^{9}$. Lymph node data (290b, 298c, 302c) are shared with James et al. and were processed with additional flow sorting 9 . 
Patients who donated diseased tissue for scRNAseq were enrolled in the 'Investigating how childhood tumors and congenital disease develop' study (NHS National Research Ethics Service (16/EE/0394)).

\section{Single cell RNA sequencing analysis}

Single-cell RNA sequencing (scRNAseq) data from cHL specimens were kindly shared by the Stiedl lab (University of British Columbia) as a raw counts matrix ${ }^{10}$. Mapping and quantification of scRNAseq generated for this study ('WSI dataset') was performed using kallisto-bustools $(0.24 .4)^{11}$, against the GRCh38 human reference. The unfiltered count matrix was profiled using defaultDrops in the DropletUtils package $(1.10 .3)^{12}$, in $\mathrm{R}(4.0 .4)$.

We performed data integration with batch correction (using donor as the batch key) and dimensionality reduction concurrently by training a single-cell variational inference model using the scvi-tools python package $(0.9 .0 \mathrm{a} 0)^{13}$. We completed quality control on the integrated dataset (Supplementary methods, Supplementary Fig. 1a-b). A 15-dimensional latent representation was used as input to nearest neighbor graph construction, Uniform Manifold and Approximation (UMAP), and Leiden clustering in scanpy. Marker genes were calculated using the tf-idf metric and genes were ranked by the tf-idf metric, and $p$ values calculated by a hypergeometric test, corrected for multiple testing (Benjamini-Hochberg method $)^{14}$. Cell type annotation was performed using marker genes, canonical marker expression (Supplementary Fig. 1c), supplemented with labeling using the celltypist python package $(0.1 .15)^{15}$. Concordance between annotated cell-type labels and predicted reference annotations was assessed by calculating the Jaccard distance between cell label strings (Supplementary Fig. 1d-e). Cell-type predictions between the King et al. B cell atlas ${ }^{16}$ was calculated by training a multilayer perceptron classifier on the reference data and calculating predictions on unseen test data, using the scikit-learn package $(0.24 .2)^{17}$.

Relative abundance analysis was performed using the MiloR package $(0.99 .1)^{18}$. The annotated dataset was represented as a $\mathrm{k}=30$ nearest neighbor graph calculated in $\mathrm{scVI}$ latent space. This graph representation was partitioned into 22,573 overlapping neighborhoods, and cell counts per 10X channel were calculated per neighborhood. Differential abundance between disease and health was calculated using the testNhoods function, setting the design as center + disease, and norm.method=TMM.

Ligand receptor analysis was performed using CellPhoneDB $(2.1 .7)^{19}$. The count matrix was normalized to $1 \mathrm{e} 4$ total counts before input to the python implementation of CellPhoneDB which was run with -- 
iterations $=1000$, - -threshold $=0.1$, and - result-precision $=3$.

Microarray data analysis

The GEO accessions GSE39133 and GSE17920 were downloaded using the GEOquery R package $(2.60 .0)^{20}$.

For GSE39133, we performed differential expression between GC and HRSC samples using limma $(3.48 .1)^{21}$ and derived genesets by retaining genes with an absolute log fold change $>3$ and adjusted $p$ value $<0.01$.

For GSE17920, we performed WGCNA using CEMiTool (1.20.0) ${ }^{22}$, and annotated these modules using enrichment analysis against cell-type marker gene sets and Gene Ontology terms using ClusterProfiler $(4.4 .4)^{23}$.

Transcription factor regulon and signaling network analysis

Transcription factor regulons were acquired using the dorothea $\mathrm{R}$ package (1.3.3) ${ }^{24}$. Regulons with confidence levels "A-C" were used to calculate transcription factor activities using run_viper.

We derived a network graph of transcription factors and targets or ligands and receptors using data from the OmnipathR R package $(3.12)^{25}$, plotting the interaction networks using the ggraph R package (2.0.5).

Tissue samples for Nanostring and Multiplexed Immunofluorescence

The study was approved by a UK NHS Health Research Authority Research Tissue Bank (Novopath Biobank, Newcastle upon Tyne Hospitals NHS Foundation Trust (17/NE0070)). Novopath released linkanonymized, formalin-fixed and paraffin embedded (FFPE) tissue, from patients with cHL. Tissue samples were surplus to diagnostic requirements and were released in accordance with the terms of the ethical approval.

FFPE biopsies (all excised lymph nodes) were selected from the pathology archives of Newcastle upon Tyne Hospitals NHS Foundation Trust, by members of Novopath, with appropriate ethical approval (17/NE0070). Limited, link-anonymized clinical data for study patients were collected by the biobank. Hematoxylin and eosin-stained (H\&E) and immunohistochemical tissue sections were reviewed by a hematologist, together with original pathology reports. Fifty-four tumors were selected for the study, 
including biopsies from patients of all ages and histomorphological subtypes (Table 2). Patient biopsies were selected on the basis of high-quality, whole lymph node resection biopsy tissue. Patients with coexisting malignant diagnoses were excluded from the study, even if these were not apparent in the tissue.

\section{Nanostring spatial transcriptomics}

Ten of 54 FFPE cHL tissue biopsies used in the multiplexed immunofluorescence analysis were selected for in situ transcriptomics (Table 2), using the Nanostring GeoMx platform (Nanostring, Seattle, WA). Biopsies were selected based upon year of fixation (2014-2019), high quality multiplex staining, and successful RNAscope quality control (QC) tests (Advanced Cell Diagnostics, Inc., Newark, CA). Five test slides were prepared, each arrayed with 2 unique, whole tissue biopsy sections; one slide also included reactive lymph node tissue. For each test slide, 4 consecutive 4 - $\mu$ m-thick sections were taken from each tissue block, prepared according to manufacturer's instructions: Slide 1, Hematoxylin \& Eosin; Slide 2, CD45 and PAX5 immunostains (fluorescent IHC); Slide 3, CD274 (PD-L1) and PDCD1 (PD-1) - RNAscope probes, DNA counterstain; Slide 4, Oligonucleotide RNA probe hybridization.

Circular regions of interest (ROIs; uniform $300 \mu \mathrm{m}$ diameter) were labeled upon the CD274/PDCD1 (PDL1) RNAscope slides, with reference to H\&E and IHC slides for histomorphological context. PD-L1 was used to identify HRSC, rather than CD30, as fluorescent labeling with $C D 274$ probes was more robust in optimization experiments. In all selected tumors, the HRSC-dense areas of tissue were known to be PD$\mathrm{L} 1^{+}$, and HRSC were identified by DAPI signal and nuclear morphology. For each tumor, 3 ROIs were

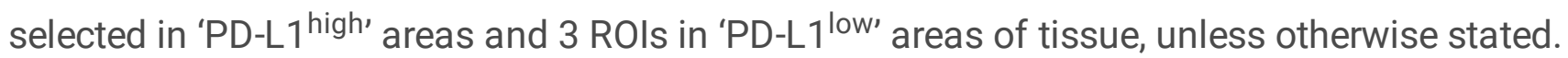
Assessments of PD-L1 intensity were made by visual inspection of normalized CD274 probe fluorescent signal within each tumor. Sclerotic bands, germinal centers, and areas of low cellularity were avoided in $\mathrm{CHL}$ tissues, by referring to DNA counterstaining and paired IHC. In the control RN tissue, 3 ROls were labeled in germinal centers and 3 ROls in interfollicular areas. RNA oligonucleotides from the 'Cancer Transcriptome Atlas' ( $\mathrm{n}=1812$ probes), coupled to photocleavable oligonucleotide tags ('barcodes'), were hybridized overnight. Hybridized barcodes were released from tissue by UV exposure from each ROI sequentially, before being counted and sequenced on the Illumina HiSeq 2500 platform.

\section{Nanostring data analysis}

Quality control excluded outlier probes, and data were normalized against the 75th percentile of signal from each tumor. Following QC, 1372 (75.7\%) probes were retained. The nanostring expression matrix (ROI-by-gene) was analyzed by principal component analysis (PCA) using scanpy, before constructing a 
shared-nearest neighbor graph representation. We identified $\mathrm{k}=10$ nearest neighbors in PCA space using the FNN R package, before calculating the Jaccard distance between neighbor vectors after the approach of Levine et $a^{26}$. The graph was clustered using the Leiden algorithm ${ }^{27}$ with default settings.

Deconvolution was performed by first generating a matrix of one-hot-encodings of marker genes per celltype, including HRSC. We then used the $r / m$ function in the MASS R package to perform robust linear regression. The resulting coefficients were treated as fractions to derive counts by multiplying by the number of nuclei in each ROI. These cell count estimates were then used to calculate differential abundance estimates for each cluster using DESeq $2^{28}$. Differential expression analysis between clusters was performed using limma (3.48.1) $)^{21}$. The top 10 differentially expressed genes per cluster were selected for plotting (Supplementary Fig. 3c)

\section{Multiplexed immunofluorescence}

Multiplexed immunofluorescence (IF) was performed by sequentially immunostaining $4 \mu \mathrm{m}$-thick tissue sections from selected FFPE biopsies, with primary antibodies, secondary reagents, and unique fluorochromes. Each of the two multiplexes comprises 6 primary antibodies, followed by a nuclear counterstain, 4',6-diamidino-2-phenylindole, as per published protocols ${ }^{7,29,30}$. All immunostaining was performed on the automated Ventana Benchmark platform (Roche Diagnostics, Rotkreuz, Switzerland). Slides were air dried, mounted with Prolong Diamond anti-fade mounting medium (\#P36965; Life Technologies) and stored at $4^{\circ} \mathrm{C}$ in a light proof box until image acquisition. Target antigens, antibody clones, and dilutions for markers are listed in Table 3.

\section{Image acquisition}

Test regions for multiplex IF analysis were initially identified for each tumor biopsy $(n=54)$ by reviewing $\mathrm{H} \& \mathrm{E}$ and immunofluorescence tissue sections (4x magnification). Three non-overlapping regions of interest were then acquired at 20x magnification for each multiplex panel. Each of these 3 test regions comprised 9 contiguous fields of view (FOVs), arranged as a $3 \times 3$ rectangular grid (measuring $2008 \mu \mathrm{m} \mathrm{x}$ $1508 \mu \mathrm{m}$ in total). There were a total of 27 FOVs per tumor, for each multiplex.

Regions were selected to exclude tissue artifacts, best represent the overall tissue, and to include CD30 ${ }^{+}$ HRSCs. Regions were imaged and deconvoluted using the Vectra multispectral imaging platform (Vectra 3, Inform 2.4.8, Akoya Biosciences, Marlborough, MA), using specific spectral libraries.

\section{Results}


To overview the cellular ecosystem of $\mathrm{cHL}$, we compiled a census of 243,753 single cell transcriptomes from lymphoma-affected and unaffected lymph nodes. We sourced data from Aoki et al ${ }^{10}$ incorporating droplet encapsulation single cell transcriptomes (10X genomics) from reactive and cHL nodes (Fig. 1a). Additionally, we performed scRNAseq (10X genomics) with cell suspensions from lymph nodes from deceased organ donors, and two lymphoma lymph nodes, one with nodular sclerosis $\mathrm{CHL}$ (NSCHL), and one with nodular lymphocyte predominant Hodgkin lymphoma (NLPHL), a biologically distinct subtype of Hodgkin lymphoma. The combined dataset comprises tissue from 13 non-lymphoma donors (8 deceased donors, 5 donors with reactive lymph node hyperplasia), one NLPHL, and $23 \mathrm{cHL}$ lymph nodes (Fig. 1a, Table 4).

After quality control (Supplementary Methods, Supplementary Fig. 1a-b), we performed dataset integration and dimensionality reduction using single-cell variational inference ${ }^{13}$ and annotated cell-types on the basis of marker genes and external dataset validation (Fig. 1b, Supplementary Fig. 1c-e).

The cellular ecosystem of $\mathrm{cHL}$ encompassed subsets of $\mathrm{CD} 4^{+}$and $\mathrm{CD} 8^{+} \mathrm{T}$-cells, including regulatory $\mathrm{T}$ cells (Treg), $T$ follicular helper cells (Tfh), and exhausted CD4 ${ }^{+}$(ThExh) and CD $8^{+}$T-cells (CD8 TExh) cells (Fig. 1b). ThExh matched the recently identified $\mathrm{LAG}^{+}$subset, and expressed checkpoint molecules and exhaustion markers including CD27, TNFRSF18, LAG3, and ICOS ${ }^{10,31}$ (Supplementary Fig. 1f). The B cell compartment split into two clusters of memory and naive subsets, in addition to plasmablasts, and germinal center (GC) B cells (Fig. 1b, Supplementary Fig. 1c-e).

The inclusion of non-lymphoma lymph nodes, including healthy, non-reactive tissue, allowed us to robustly test the differential abundance of cells between lymphoma and 'non-lymphoma', using a neighborhood partitioning approach (MiloR - Methods) ${ }^{18}$. Lymphoma was enriched for cytotoxic lymphocyte subsets including effector memory CD8+ T-cells (CD8 Tem) and NK cells, in addition to CD4+ T-cell subsets expressing checkpoint apparatus, including ThExh and Tfh cells (Fig. 1f, Supplementary Fig. 2b). Within the lymphoma samples, $\mathrm{CHL}$ was enriched for exhausted T-cell subsets, in contrast with the NLPHL sample which was dominated by cytotoxic subsets (NK cells, CD8 Tem, and CD8 Tcm) (Supplementary Fig. 2c).

We did not detect HRSC, likely due to the rarity, size, and fragility of these cells. To probe their transcriptional programs, which potentially orchestrate this immunoregulatory milieu, we leveraged a microarray dataset profiling microdissected HRSC and GCs (Methods) ${ }^{32}$. Differential expression between HRSC and GC identified an HRSC gene signature, including TNFRSF8 (CD30) (Fig. 1g). Scoring of 
transcription factor regulons demonstrated NF-KB activation (NFKB1 and its activatory heterodimer partner RELA) in HRSC (Fig. 1h), consistent with previous reports ${ }^{33}$. We next performed in silico identification of interactions between active transcription factors and potential targets within the HRSC geneset (Methods). This demonstrated an NF-KB-centric network coordinating the upregulation of the chemokines CCL5, CCL17, and CCL22 capable of the positioning and retention of ThExh via CCR5 and CCR4 ligation (Fig. 1i, Supplementary Fig. 1f).

Within the myeloid compartment, we identified macrophages coexpressing $C D 14, C D 68$, and resident macrophage markers FOLR2 and MRC1 (Fig. 1c-d). These cells were transcriptionally distinct from classical monocytes, which expressed a characteristic signature including S100A9, CD14, $V C A N$, and $F C N 1^{34}$ (Fig. 1c-d). Amongst DCs, we identified CDC1 (key transcripts: CLEC9A, CADM1, IDO1) and CDC2 (key transcripts: CD1C, CLEC1OA, FCER1A) (Fig. 1c-d). We also identified LAMP3' DCs in both healthy and some lymphoma samples, expressing the chemokine receptor $C C R 7$ and the chemokines CCL17 and CCL19, which we termed "activated DC" (aDC), consistent with the nomenclature of transcriptionally similar cells described in human thymus and spleen ${ }^{35,36}$, and in murine lung neoplasms ${ }^{37}$ (Fig. 1c-d, Supplementary Fig. 1d). We found a population of plasmacytoid DC (pDC) with a dominant contribution from lymphoma samples (Fig. 1e, Supplementary Fig. 2a), expressing IL3RA (CD123) and CLEC4C (Fig. 1c-d).

Within the stromal cell compartment, we identified fibroblasts (key transcripts: $\angle U M, D C N$ ), and endothelial cells (key transcripts: CLDN5, PECAM1, and PLVAP (fenestrated endothelium)) (Fig. 1b, Supplementary Fig. 1c).

We used this lymph node-wide account of the immune landscape of $\mathrm{cHL}$ as a reference to interrogate the cellular composition of cHL tissues, focusing on myeloid cells.

We first identified spatially distinct microenvironments using targeted spatial transcriptomic profiling (Nanostring GeoMx Cancer Transcriptome Atlas), defining $300 \mu \mathrm{m}$ diameter regions of interest (ROI) in both PD-L $1^{\text {high }}$ and PD-L $1^{\text {low }}$ regions of $9 \mathrm{NSCHL}$ and 1 Mixed Cell $\mathrm{CHL}$ (MCCHL) lymph nodes and follicular and interfollicular regions of one control reactive lymph node (Supplementary Fig. 3a-b). We represented these transcriptional profiles in a shared-nearest neighbor graph and identified 5 clusters (Fig. 2a-b). We then deconvoluted the cell composition of each cluster using our scRNAseq reference, followed by cell-type count estimation and differential cell-type abundance analysis (Fig. 2c, Methods). The clusters encompassed two 'neoplastic' PD-L1 high HRSC-enriched clusters (clusters 3 \& 4), two 'non- 
neoplastic' PD-L1 ${ }^{\text {low }}$ HRSC-depleted clusters (clusters 1 \& 5), and one intermediate neighborhood (cluster 2).

Clusters 1 and 5 were PD-L $1^{\text {low }}$ and represented fibrotic regions and healthy follicular microenvironments respectively. Cluster 1 was enriched for stromal (fibroblast and endothelial) cells, classical monocytes and pDC, but was depleted of CDC (Fig. 2C). Differentially expressed genes in this cluster included genes encoding signaling mediators of fibrosis FGFR4, TGFB2, and PTCH1 (Supplementary Fig. 3c). In contrast cluster 5 was exclusively derived from control samples and enriched for GC B cells, Tfh, and plasmablasts (Fig. 2a-c).

The PD-L $1^{\text {high }}$ neoplastic clusters exhibited divergent leukocyte enrichment. Cluster 3 was enriched for ThExh, Th, and NK cells. In contrast, cluster 4 represented a myeloid-enriched niche, characterized classical monocyte, macrophage, and CDC2 infiltration (Fig. 2c). Marker genes for cluster 4 highlighted inflammatory signaling, with upregulation of chemokines associated with Th2 responses and CCR3dependent eosinophil recruitment (CCL18, CCL13, CCL24, CCL26, CCL23) and granulocyte-attracting chemokines CXCL1, and CXCL6 (Supplementary Fig. 3c).

We next sought to identify transcriptional correlates of these microenvironments in publicly available gene expression data. We performed Weighted Correlation Network Analysis (WGCNA) using data from 130 diagnostic $\mathrm{CHL}$ lymph nodes ${ }^{22,38}$. This analysis yielded 6 modules of co-expressed genes (modules A-F) (Fig. 2d). We performed enrichment analysis using both scRNAseq references and Gene Ontology terms to annotate these modules and found that whilst module $\mathrm{C}$ corresponds to the cell cycle program (Supplementary Fig. 3d), the other modules strikingly mirror the tissue niche patterns identified in our spatially resolved (Nanostring) transcriptomics data (Fig. 2d), suggesting a highly conserved organization of pathological niches in $\mathrm{CHL}$.

Module A enriched for stromal cell signatures similarly to PD-L $1^{\text {low }}$ nanostring cluster 1 . In contrast, module $B$ represented myeloid-skewed inflammation, enriching for monocyte, macrophage, and cDC2 signatures, aligning to nanostring cluster 4 (Fig. 2d).

We then interrogated the relationship between module gene expression and treatment outcomes. High expression of the fibrosis/stroma enriched module was associated with treatment success, whereas high 
expression of the myeloid enriched module was associated with early treatment failure. Expression of module F (B cell follicle) was not associated with differences in outcome (Fig. 2d).

Given variation in microenvironmental myeloid infiltration appears to be a conserved and prognostically important feature of $\mathrm{CHL}$ pathobiology, we sought to define MNP within the HRSC niche at single-cell resolution. Using marker gene co-expression patterns in the scRNAseq data (Supplementary Fig. 3e), we designed multiplexed immunofluorescence panels to identify MNPs in cHL tissue sections ( $n=54$ tumors, representative images: Fig. 2e-g). We identified CADM1 ${ }^{+} / \mathrm{CD} 11 \mathrm{c}^{+} \mathrm{cDC} 1, \mathrm{CD} 1 \mathrm{c}^{+} / \mathrm{CD} 11 \mathrm{c}^{+} \mathrm{cDC} 2, \mathrm{LAMP}^{+}$

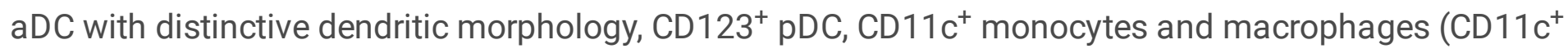
$\mathrm{ONLY}$ ), and CD30+ HRSC (Fig. 2d). We then phenotyped segmented cells and performed neighborhood analyses, taking a $25 \mu \mathrm{m}$ radius neighborhood around each $\mathrm{CD} 30^{+} \mathrm{HRSC}$ and measuring the relative enrichment of MNP subsets in aggregated neighborhoods, compared to 'non-neighborhood' regions (Fig. 2f). This revealed enrichment of $\mathrm{CDC} 2$ and $C D 11 \mathrm{c}^{+}$monocytes in the immediate vicinity of HRSC. In contrast, both $\mathrm{pDC}$ and $\mathrm{aDC}$ were excluded from the HRSC niche and occupy regions with a low density of $\mathrm{CD} 11 \mathrm{c}^{+}$cells (Fig. 2e-h, Fig. 3a-h).

We next asked which signals might coordinate the positioning of MNP subsets and T-cells found in close association with HRSC. To interrogate ligand-receptor interactions (LRI), we calculated the statistical enrichment of candidate LRI between MNPs and T-cells in our scRNAseq data, using the CellPhoneDB tool $^{19}$. This analysis predicted paracrine CCL3 and CCL4 signaling by macrophages and classical monocytes to CCR5- and CCR1-expressing CDC2 and ThExh (Fig. 4a). Furthermore, classical monocytederived CXCL10 was predicted to signal to CDC1, ThExh, and Tfh via CXCR3. Nominated inhibitory interactions from ThExh included TIGIT signaling via NECTIN2 expressed by cDC2, classical monocytes, and macrophages (Fig. 4a). Analysis of putative LRI interactions inferred from Nanostring cluster 4 and WGCNA module B also highlighted CCR1-and CCL5-mediated signaling (Supplementary Fig. 4a). A proposed schematic illustrates potential interactions between HRSC, CDC2, classical monocytes, and TExh (Fig. 4b).

We were surprised that the HRSC-associated MNP network includes CDC2 and occasional CDC1, which play key roles in immunosurveillance. Although macrophage expression of PD-L1 is established, upregulation of inhibitory ligands and receptors by $\mathrm{DC}$ has not been described in $\mathrm{CHL}$. To test this, we designed a second IF panel to examine expression of PD-L1, ID01, and TIM-3 on CD11 $\mathrm{C}^{+} / \mathrm{CD}^{-} 8^{-} \mathrm{CDC} 1$ and $\mathrm{CDC} 2, \mathrm{CD} 11 \mathrm{c}^{+} / \mathrm{CD} 68^{+}$monocytes, and $\mathrm{CD} 11 \mathrm{c}^{-} / \mathrm{CD} 68^{+}$macrophages, and to assess their spatial relationships to $\mathrm{CD}^{3} 0^{+} \mathrm{HRSC}$ (Table 3 ). 
HRSC exhibited extensive PD-L1 expression as expected. We also found enrichment of PD$\mathrm{L}^{+}{ }^{+} \mathrm{CD} 11 \mathrm{c}^{+} / \mathrm{CD}^{+} 8^{+}$and $\mathrm{CD} 11 \mathrm{c}^{+} / \mathrm{CD} 68^{-}$MNPs in $\mathrm{CD} 30^{+} \mathrm{HRSC}$ neighborhoods (Fig. 5a-d). Similarly, we found variable co-expression of the co-inhibitory receptor TIM-3, and IDO1 - an immunomodulating tryptophan catabolizing enzyme - on $\mathrm{CD} 11 \mathrm{c}^{+} / \mathrm{CD} 68^{+}$and $\mathrm{CD} 11 \mathrm{c}^{+} / \mathrm{CD} 68^{-}$MNPs in close proximity to HRSC (Fig. 5a-e, Supplementary Fig. 5).

The proportion of $\mathrm{CD} 11 \mathrm{c}^{+} / \mathrm{CD} 68^{-}$and $\mathrm{CD} 11 \mathrm{c}^{+} / \mathrm{CD} 68^{+}$MNPs expressing inhibitory molecules increased with age at diagnosis $(\mathrm{p}<0.05)$, but there were no significant differences in the proportion of MNPs expressing inhibitory molecules according to sex, histological subtype, or EBV status (Supplementary Fig. 6). This finding suggests immunosuppressive signaling in pediatric and young-adult $\mathrm{CHL}$ may differ from $\mathrm{cHL}$ in older adults.

\section{Discussion}

HRSC have the remarkable ability to cohere an immunosuppressive microenvironment, including the recruitment of MNP expressing immunoregulatory molecules. Through high-resolution analysis, we find that MNP comprise diverse subsets of monocytes, macrophages, and DC. We highlight several new features of this MNP network: the exclusion of $\mathrm{pDC}$ and $\mathrm{aDC}$ from the vicinity of HRSC; that immunoregulatory molecules are present on DC close to HRSC, at equivalent levels to macrophages; that multiple soluble and cell-cell interactions underpin this structure, communicating with HRSC and lymphocytes. This work complements recent detailed analysis of lymphocytes in $\mathrm{cHL}$, showing enrichment of exhausted LAG3 ${ }^{+} \mathrm{CD}^{+}{ }^{+}$T-cells around MHC-II- HRSC ${ }^{10}$, and elaboration of CXCL13 by Tfhlike cells in lymphocyte-rich $\mathrm{CHL}^{31}$.

We have shown that conventional DC are closely associated with PD-L $1^{+} \mathrm{HRSC}$. These cells are usually involved in immunosurveillance and presentation of neoantigens to T-cells, however this mechanism appears to be stalled in $\mathrm{CHL}$, likely as a result of immunosuppressive niche signaling. Colocalization and LRI analyses suggest that MNP have proximal roles in directing and amplifying this signaling. These MNP cooperate with HRSC-derived and NF-KB-directed chemokine expression, providing chemoattractant and inhibitory signals that recruit and instruct immunosuppressive T-cells. Our analysis identifies classical monocytes in particular as important signaling hubs, controlling retention of CDC2 and ThExh via CCR1-, CCR4-, CCR5-, and CXCR3-dependent signaling (Fig. 4a-b). 
We propose that there are distinct and recurring tissue niches within $\mathrm{cHL}$ lymph nodes. Enrichment for the inflammatory cDC2-monocyte-macrophage niche is associated with early relapse following treatment, corroborating previous reports that $\mathrm{CD} 68^{+} \mathrm{TAM}$ infiltration and macrophage gene expression is associated with poor outcomes in advanced $\mathrm{cHL}^{38,39}$. Conversely, a fibrosis/stroma-rich tissue niche is associated with favorable outcomes. Computational pathology approaches and high-throughput multiplexed may permit the characterization of these diverging cell networks in diagnostic tissue sections technologies ${ }^{40,41}$. Future strategies might aim to shift the dominant pathology towards a fibrotic or MNPdepleted phenotype (e.g. CCL2 inhibition, CSF-CSF1R blockade), and away from an inflammatory and immunosuppressive phenotype ${ }^{42-44}$. DC may be augmented by Flt3-ligand ${ }^{45}$, CD40 agonists, or lentivirally-transduced chimeric antigen receptors ${ }^{46}$.

Therapeutic efforts to reverse tumor-associated immune suppression have targeted T-cells, with recent studies suggesting alternative immune checkpoints, in addition to PD-1 ${ }^{10,47}$. The co-expression of inhibitory molecules and chemokines across MNP suggests extensive redundancy in immunosuppressive signaling ${ }^{48,49}$. This may partially explain resistance to PD-1 blockade and offer opportunities for tailoring therapies on the basis of molecular profiling. Intriguingly, a recent report demonstrated that HRSC eradication, following induction Nivolumab, was associated with a reduction in PD-L $1^{+} \mathrm{TAM}$, rather than detectable cytotoxic responses ${ }^{50}$. This suggests initial vulnerability of MNP networks to PD-1 blockade, such as dependence on tonic signaling by T-cells or HRSC, and may support the hypothesis of reverse signaling on $\mathrm{HRSC}^{51}$.

Overall, these atlas-scale molecular and spatially resolved data provide evidence for distinctly organized tissue niches defining the pathology of $\mathrm{cHL}$. Our results highlight a dominant, interacting and immunosuppressive MNP network associated with HRSC, the rational targeting of which may present valuable therapeutic opportunities.

\section{Declarations}

\section{Acknowledgements}

BJS is supported by a Clinical Doctoral Fellowship from the Wellcome Trust (216366/Z/19/Z).

CDC is supported by grant funding from the JGW Patterson Foundation (https://jgwpattersonfoundation.co.uk), Bright Red (https://brightred.org.uk), the Lymphoma Research Trust, a Medical Research Council 'Confidence in Concepts' award, and a Wellcome Trust Small project grant award. 
We thank Dr Christian Stiedl (The University of British Columbia) for generous sharing of scRNAseq data. We thank the Newcastle University / Newcastle upon Tyne NHS Foundation Trust Molecular diagnostics laboratory (NovoPath; https://www.novopath.co.uk/) for tissue handling and technical assistance.

\section{Author contributions}

BJS performed data analysis and wrote the manuscript, MF performed data analysis and contributed to the manuscript, MDY contributed to data curation, CJ, AS, AB, CB, VR, JRF performed scRNAseq experiments, KTM and KSP acquired deceased donor lymph node tissue, MRC and SB supervised the project, CDC performed microscopy experiments, analyzed data, wrote the manuscript, and supervised the project.

\section{Conflict of interest}

None declared

\section{Code availability}

Code used to analyze data in the project is uploaded to Github at https://github.com/bjstewart1/hodgkin_lymphoma_analysis

\section{Data availability}

Data is made available to download on this website as an annotated data object (.h5ad file) and interactive cellxgene portal.

\section{References}

1. Sasse S, Bröckelmann PJ, Goergen H, et al. Long-Term Follow-Up of Contemporary Treatment in Early-Stage Hodgkin Lymphoma: Updated Analyses of the German Hodgkin Study Group HD7, HD8, HD10, and HD11 Trials. J. Clin. Oncol. 2017;35(18):1999-2007.

2. Borchmann $\mathrm{P}$, Goergen $\mathrm{H}$, Kobe $\mathrm{C}$, et al. PET-guided treatment in patients with advanced-stage Hodgkin's lymphoma (HD18): final results of an open-label, international, randomised phase 3 trial by the German Hodgkin Study Group. Lancet. 2017;390(10114):2790-2802. 
3. Casasnovas R-O, Bouabdallah R, Brice P, et al. PET-adapted treatment for newly diagnosed advanced Hodgkin lymphoma (AHL2011): a randomised, multicentre, non-inferiority, phase 3 study. Lancet Oncol. 2019;20(2):202-215.

4. Connors JM, Cozen W, Steidl C, et al. Hodgkin lymphoma. Nat Rev Dis Primers. 2020;6(1):61.

5. Roemer MGM, Redd RA, Cader FZ, et al. Major Histocompatibility Complex Class II and Programmed Death Ligand 1 Expression Predict Outcome After Programmed Death 1 Blockade in Classic Hodgkin Lymphoma. J. Clin. Oncol. 2018;36(10):942-950.

6. Green MR, Monti S, Rodig SJ, et al. Integrative analysis reveals selective 9p24.1 amplification, increased PD-1 ligand expression, and further induction via JAK2 in nodular sclerosing Hodgkin lymphoma and primary mediastinal large B-cell lymphoma. Blood. 2010;116(17):3268-3277.

7. Carey CD, Gusenleitner D, Lipschitz M, et al. Topological analysis reveals a PD-L1-associated microenvironmental niche for Reed-Sternberg cells in Hodgkin lymphoma. Blood. 2017;130(22):24202430 .

8. Mulder K, Patel AA, Kong WT, et al. Cross-tissue single-cell landscape of human monocytes and macrophages in health and disease. Immunity. 2021;54(8):1883-1900.e5.

9. James KR, Gomes T, Elmentaite R, et al. Distinct microbial and immune niches of the human colon. Nat. Immunol. 2020;21(3):343-353.

10. Aoki T, Chong LC, Takata K, et al. Single-Cell Transcriptome Analysis Reveals Disease-Defining T-cell Subsets in the Tumor Microenvironment of Classic Hodgkin Lymphoma. Cancer Discov. 2020;10(3):406421.

11. Melsted P, Ntranos V, Pachter L. The barcode, UMI, set format and BUStools. Bioinformatics. 2019;35(21):4472-4473.

12. Lun ATL, Riesenfeld S, Andrews T, et al. EmptyDrops: distinguishing cells from empty droplets in droplet-based single-cell RNA sequencing data. Genome Biol. 2019;20(1):63.

13. Lopez R, Regier J, Cole MB, Jordan MI, Yosef N. Deep generative modeling for single-cell transcriptomics. Nat. Methods. 2018;15(12):1053-1058.

14. Young MD, Mitchell TJ, Vieira Braga FA, et al. Single-cell transcriptomes from human kidneys reveal the cellular identity of renal tumors. Science. 2018;361(6402):594-599.

15. Domínguez CC, Gomes T, Jarvis LB, et al. Cross-tissue immune cell analysis reveals tissue-specific adaptations and clonal architecture across the human body. 
16. King HW, Orban N, Riches JC, et al. Single-cell analysis of human B cell maturation predicts how antibody class switching shapes selection dynamics. Science Immunology. 2021;6(56):eabe6291.

17. Garreta R, Moncecchi G. Learning scikit-learn: Machine Learning in Python. Packt Publishing Ltd; 2013.

18. Dann E, Henderson NC, Teichmann SA, Morgan MD, Marioni JC. Differential abundance testing on single-cell data using k-nearest neighbor graphs. Nat. Biotechnol. 2021;

19. Efremova M, Vento-Tormo M, Teichmann SA, Vento-Tormo R. CellPhoneDB: inferring cell-cell communication from combined expression of multi-subunit ligand-receptor complexes. Nat. Protoc. 2020;15(4):1484-1506.

20. Davis S, Meltzer PS. GEOquery: a bridge between the Gene Expression Omnibus (GEO) and BioConductor. Bioinformatics. 2007;23(14):1846-1847.

21. Ritchie ME, Phipson B, Wu D, et al. limma powers differential expression analyses for RNAsequencing and microarray studies. Nucleic Acids Res. 2015;43(7):e47.

22. Russo PST, Ferreira GR, Cardozo LE, et al. CEMiTool: a Bioconductor package for performing comprehensive modular co-expression analyses. BMC Bioinformatics. 2018;19(1):56.

23. Wu T, Hu E, Xu S, et al. clusterProfiler 4.0: A universal enrichment tool for interpreting omics data. Innovation (Camb). 2021;2(3):100141.

24. Garcia-Alonso L, Holland CH, Ibrahim MM, Turei D, Saez-Rodriguez J. Benchmark and integration of resources for the estimation of human transcription factor activities. Genome Res. 2019;29(8):13631375.

25. Türei D, Korcsmáros T, Saez-Rodriguez J. OmniPath: guidelines and gateway for literature-curated signaling pathway resources. Nat. Methods. 2016;13(12):966-967.

26. Levine JH, Simonds EF, Bendall SC, et al. Data-Driven Phenotypic Dissection of AML Reveals Progenitor-like Cells that Correlate with Prognosis. Cell. 2015;162(1):184-197.

27. Traag VA, Waltman L, van Eck NJ. From Louvain to Leiden: guaranteeing well-connected communities. Sci. Rep. 2019;9(1):5233.

28. Love MI, Huber W, Anders S. Moderated estimation of fold change and dispersion for RNA-seq data with DESeq2. Genome Biol. 2014;15(12):550.

29. Feng Z, Puri S, Moudgil T, et al. Multispectral imaging of formalin-fixed tissue predicts ability to generate tumor-infiltrating lymphocytes from melanoma. J Immunother Cancer. 2015;3:47. 
30. Tóth ZE, Mezey E. Simultaneous visualization of multiple antigens with tyramide signal amplification using antibodies from the same species. J. Histochem. Cytochem. 2007;55(6):545-554.

31. Aoki T, Chong LC, Takata K, et al. Single-cell profiling reveals the importance of CXCL13/CXCR5 axis biology in lymphocyte-rich classic Hodgkin lymphoma. Proc. Natl. Acad. Sci. U. S. A. 2021;118(41.):

32. Steidl C, Diepstra A, Lee T, et al. Gene expression profiling of microdissected Hodgkin Reed-Sternberg cells correlates with treatment outcome in classical Hodgkin lymphoma. Blood. 2012;120(17):35303540 .

33. Nagel D, Vincendeau M, Eitelhuber AC, Krappmann D. Mechanisms and consequences of constitutive NF-KB activation in B-cell lymphoid malignancies. Oncogene. 2014;33(50):5655-5665.

34. Villani A-C, Satija R, Reynolds G, et al. Single-cell RNA-seq reveals new types of human blood dendritic cells, monocytes, and progenitors. Science. 2017;356(6335.):

35. Park J-E, Botting RA, Domínguez Conde $C$, et al. A cell atlas of human thymic development defines T cell repertoire formation. Science. 2020;367(6480.):

36. Madissoon E, Wilbrey-Clark A, Miragaia RJ, et al. scRNA-seq assessment of the human lung, spleen, and esophagus tissue stability after cold preservation. Genome Biol. 2019;21(1):1.

37. Maier B, Leader AM, Chen ST, et al. A conserved dendritic-cell regulatory program limits antitumour immunity. Nature. 2020;580(7802):257-262.

38. Steidl C, Lee T, Shah SP, et al. Tumor-associated macrophages and survival in classic Hodgkin's lymphoma. N. Engl. J. Med. 2010;362(10):875-885.

39. Tan KL, Scott DW, Hong F, et al. Tumor-associated macrophages predict inferior outcomes in classic Hodgkin lymphoma: a correlative study from the E2496 Intergroup trial. Blood. 2012;120(16):3280-3287.

40. Chen RJ, Lu MY, Williamson DFK, et al. Pan-cancer integrative histology-genomic analysis via multimodal deep learning. Cancer Cell. 2022;40(8):865-878.e6.

41. Kuett L, Catena R, Özcan A, et al. Three-dimensional imaging mass cytometry for highly multiplexed molecular and cellular mapping of tissues and the tumor microenvironment. Nat Cancer. 2022;3(1):122133.

42. Cassetta L, Pollard JW. Targeting macrophages: therapeutic approaches in cancer. Nat. Rev. Drug Discov. 2018;17(12):887-904.

43. Teng K-Y, Han J, Zhang X, et al. Blocking the CCL2-CCR2 axis using CCL2-neutralizing antibody is an effective therapy for hepatocellular cancer in a mouse model. Mol. Cancer Ther. 2017;16(2):312-322. 
44. Pyonteck SM, Akkari L, Schuhmacher AJ, et al. CSF-1R inhibition alters macrophage polarization and blocks glioma progression. Nat. Med. 2013;19(10):1264-1272.

45. Saito T, Takayama T, Osaki T, et al. Combined mobilization and stimulation of tumor-infiltrating dendritic cells and natural killer cells with Flt3 ligand and IL-18 in vivo induces systemic antitumor immunity. Cancer Sci. 2008;99(10):2028-2036.

46. Klichinsky $M$, Ruella $M$, Shestova 0 , et al. Human chimeric antigen receptor macrophages for cancer immunotherapy. Nat. Biotechnol. 2020;38(8):947-953.

47. Patel SS, Weirather $\mathrm{JL}$, Lipschitz $\mathrm{M}$, et al. The microenvironmental niche in classic Hodgkin lymphoma is enriched for CTLA-4-positive T cells that are PD-1-negative. Blood. 2019;134(23):20592069.

48. Haderk F, Schulz R, Iskar M, et al. Tumor-derived exosomes modulate PD-L1 expression in monocytes. Sci Immunol. 2017;2(13.):

49. Karihtala K, Leivonen S-K, Karjalainen-Lindsberg M-L, et al. Checkpoint protein expression in the tumor microenvironment defines the outcome of classical Hodgkin lymphoma patients. Blood Adv. 2022;6(6):1919-1931.

50. Reinke S, Bröckelmann PJ, laccarino I, et al. Tumor and microenvironment response but no cytotoxic T-cell activation in classic Hodgkin lymphoma treated with anti-PD1. Blood. 2020;136(25):2851-2863.

51. Jalali S, Price-Troska T, Bothun C, et al. Reverse signaling via PD-L1 supports malignant cell growth and survival in classical Hodgkin lymphoma. Blood Cancer J. 2019;9(3):22.

\section{Tables}

Table 1. Clinical and Pathological Characteristics of Classic Hodgkin Lymphoma Study Cases for Multiplexed Immunofluorescence and Nanostring 


\begin{tabular}{|c|c|c|c|c|c|c|c|c|}
\hline $\begin{array}{l}\text { Study } \\
\text { Tumor } \\
\text { Reference }\end{array}$ & $\begin{array}{l}\text { Disease } \\
\text { Subtype }\end{array}$ & $\begin{array}{l}\text { EBV } \\
\text { Status }\end{array}$ & GeoMx & $\begin{array}{l}\text { Age at } \\
\text { Diagnosis } \\
\text { (years) }\end{array}$ & Sex & $\begin{array}{l}\text { Site of Biopsy } \\
\text { (Excised Node) }\end{array}$ & Stage & $\begin{array}{l}\text { Clinical } \\
\text { Response }\end{array}$ \\
\hline CHL-1 & MCCHL & NEG & & 80 & M & Inguinal & $2 \mathrm{~A}$ & CR \\
\hline CHL-3 & MCCHL & NEG & & 77 & F & Submandibular & NA & CR / relapse \\
\hline CHL-4 & LRCHL & POS & & 75 & $\mathrm{~F}$ & Neck & $2 \mathrm{~A}$ & $\mathrm{CR}$ \\
\hline CHL-5 & NSCHL & NEG & & 73 & $\mathrm{~F}$ & Supraclavicular & $4 \mathrm{~B}$ & CR \\
\hline CHL-6 & NSCHL & NEG & & 73 & M & Neck & $1 \mathrm{~A}$ & CR \\
\hline CHL-7 & NSCHL & NEG & & 72 & M & Neck & $1 \mathrm{~A}$ & CR \\
\hline CHL-9 & NSCHL & NEG & YES & 69 & M & Neck & $1 \mathrm{~A}$ & $\begin{array}{c}\text { PD / } \\
\text { Salvage CR }\end{array}$ \\
\hline CHL-10 & NSCHL & NEG & YES & 69 & $\mathrm{~F}$ & Neck & 3в & $\mathrm{CR}$ \\
\hline CHL-11 & CHL-NOS & POS & & 68 & F & Neck & 3в & $\mathrm{CR} /$ relapse \\
\hline CHL-12 & CHL-NOS & POS & & 68 & $\mathrm{~F}$ & Axilla & $1 \mathrm{~A}$ & $\mathrm{CR} /$ relapse \\
\hline CHL-13 & MCCHL & NEG & & 68 & M & Inguinal & $2 \mathrm{~A}$ & $\mathrm{CR}$ \\
\hline CHL-14 & CHL-NOS & NEG & & 64 & M & Mediastinum & NA & $\begin{array}{c}\text { PD / } \\
\text { salvage CR }\end{array}$ \\
\hline CHL-15 & MCCHL & POS & & 64 & M & Neck & NA & NA \\
\hline CHL-16 & MCCHL & NEG & & 63 & F & Neck & $2 \mathrm{~A}$ & $\mathrm{CR}$ \\
\hline CHL-18 & MCCHL & POS & & 60 & $\mathrm{~F}$ & Supraclavicular & $4 \mathrm{~B}$ & CR \\
\hline CHL-19 & MCCHL & NA & & 53 & M & Inguinal & NA & $\mathrm{PD}$ \\
\hline CHL-20 & LRCHL & NA & & 46 & M & Axilla & NA & NA \\
\hline CHL-21 & MCCHL & POS & & 45 & M & Neck & $3 \mathrm{~S}$ & PD \\
\hline CHL-22 & NSCHL & POS & & 40 & M & Neck & $2 \mathrm{~A}$ & $\mathrm{CR} /$ relapse \\
\hline CHL-23 & NSCHL & POS & & 39 & M & Neck & $4 \mathrm{~A}$ & $\begin{array}{c}\mathrm{PR} / \text { salvage } \\
\text { CR }\end{array}$ \\
\hline CHL-24 & NSCHL & NA & & 38 & M & Supraclavicular & $4 \mathrm{~A}$ & $\mathrm{PD}$ \\
\hline CHL-25 & NSCHL & NEG & & 33 & $\mathrm{~F}$ & Neck & 3в & CR \\
\hline CHL-27 & NSCHL & POS & YES & 32 & M & Supraclavicular & $2 \mathrm{~A}$ & $\mathrm{PD}$ \\
\hline CHL-28 & MCCHL & POS & & 32 & M & Neck & Зв & $\mathrm{CR}$ \\
\hline CHL-29 & NSCHL & NA & & 32 & M & Neck & $4 \mathrm{~A}$ & CR / relapse \\
\hline CHL-30 & MCCHL & POS & & 28 & F & Neck & $2 \mathrm{~A}$ & CR \\
\hline Study & Disease & EBV & GeoMx & Age at & Sex & Site of Biopsy & Stage & Clinical \\
\hline
\end{tabular}




\begin{tabular}{|c|c|c|c|c|c|c|c|c|}
\hline Tumor & Subtype & Status & & $\begin{array}{c}\text { Diagnosis } \\
\text { (years) }\end{array}$ & & (Excised Node) & & Response \\
\hline CHL-31 & NSCHL & NEG & & 28 & M & Neck & $4 \mathrm{~B}$ & CR \\
\hline CHL-32 & MCCHL & POS & YES & 26 & $\mathrm{M}$ & Neck & $2 \mathrm{~A}$ & $\mathrm{CR}$ \\
\hline CHL-33 & NSCHL & POS & & 25 & M & Neck & $2 \mathrm{~B}$ & $\mathrm{CR}$ \\
\hline CHL-34 & NSCHL & NEG & YES & 24 & M & Neck & NA & NA \\
\hline CHL-36 & NSCHL & NEG & & 23 & $\mathrm{~F}$ & Neck & $2 \mathrm{~A}$ & $\mathrm{CR}$ \\
\hline CHL-39 & MCCHL & NEG & & 22 & $\mathrm{~F}$ & Supraclavicular & NA & NA \\
\hline CHL-40 & NSCHL & NEG & YES & 20 & M & Supraclavicular & 3BS & $\mathrm{CR}$ \\
\hline CHL-41 & NSCHL & NEG & & 19 & $\mathrm{~F}$ & Mediastinum & $2 \mathrm{~B}$ & $\begin{array}{c}\mathrm{PD} / \\
\text { salvage }\end{array}$ \\
\hline CHL-42 & NSCHL & NEG & & 18 & $\mathrm{~F}$ & Neck & $2 \mathrm{~B}$ & $\mathrm{PD}$ \\
\hline CHL-43 & NSCHL & NEG & YES & 18 & M & Neck & $2 \mathrm{~A}$ & $\mathrm{CR}$ \\
\hline CHL-44 & NSCHL & POS & & 18 & M & Post nasal space & $1 \mathrm{~A}$ & CR \\
\hline CHL-45 & NSCHL & NEG & & 17 & M & Neck & $2 \mathrm{~A}$ & $\begin{array}{c}\text { CR / } \\
\text { relapsed \& } \\
\text { salvage }\end{array}$ \\
\hline CHL-48 & NSCHL & NEG & & 16 & $\mathrm{~F}$ & Axilla & $3 \mathrm{~A}$ & $\begin{array}{c}\mathrm{PD} / \\
\text { salvage }\end{array}$ \\
\hline CHL-49 & MCCHL & POS & & 16 & M & Axilla & $1 \mathrm{~A}$ & $\begin{array}{c}\text { CR / } \\
\text { relapsed \& } \\
\text { salvage }\end{array}$ \\
\hline CHL-50 & NSCHL & NEG & & 16 & $\mathrm{~F}$ & Neck & $2 \mathrm{~A}$ & CR \\
\hline CHL-51 & NSCHL & POS & & 16 & $\mathrm{~F}$ & Supraclavicular & $3 \mathrm{~B}$ & $\mathrm{CR}$ \\
\hline CHL-52 & NSCHL & NA & & 15 & $\mathrm{~F}$ & Neck & $2 \mathrm{~B}$ & $\mathrm{CR}$ \\
\hline CHL-53 & CHL-NOS & NEG & & 14 & $\mathrm{~F}$ & Retroperitoneal & $3 \mathrm{~B}$ & $\mathrm{CR}$ \\
\hline CHL-54 & NSCHL & NEG & YES & 14 & M & Supraclavicular & $2 \mathrm{~A}$ & $\mathrm{CR}$ \\
\hline CHL-55 & CHL-NOS & NEG & & 14 & $\mathrm{~F}$ & Supraclavicular & $3 \mathrm{~A}$ & $\mathrm{CR}$ \\
\hline CHL-56 & NSCHL & NEG & & 14 & $\mathrm{~F}$ & Neck & $4 \mathrm{~A}$ & $\mathrm{CR}$ \\
\hline CHL-57 & NSCHL & NEG & YES & 14 & $\mathrm{M}$ & Supraclavicular & $2 \mathrm{~A}$ & $\mathrm{CR}$ \\
\hline CHL-59 & NSCHL & NEG & YES & 13 & M & Neck & $4 \mathrm{~A}$ & CR \\
\hline CHL-60 & LR-CHL & POS & & 12 & M & Neck & $1 \mathrm{~A}$ & $\mathrm{CR}$ \\
\hline CHL-61 & NSCHL & POS & & 12 & M & Neck & $2 \mathrm{~B}$ & CR \\
\hline CHL-62 & MCCHL & POS & & 8 & M & Hilar & $4 \mathrm{~A}$ & $\begin{array}{c}\mathrm{CR} / \\
\text { relapsed \& }\end{array}$ \\
\hline
\end{tabular}




\begin{tabular}{|l|c|c|c|c|c|c|c|c|} 
& & & & & & salvage \\
\hline CHL-63 & CHL-NOS & POS & & 7 & M & Neck & $4 \mathrm{~B}$ & CR \\
\hline $\begin{array}{l}\text { Study } \\
\text { Tumor }\end{array}$ & $\begin{array}{c}\text { Disease } \\
\text { Subtype }\end{array}$ & $\begin{array}{c}\text { EBV } \\
\text { Status }\end{array}$ & GeoMx & $\begin{array}{c}\text { Age at } \\
\text { Diagnosis } \\
\text { (years) }\end{array}$ & Sex & $\begin{array}{c}\text { Site of Biopsy } \\
\text { (Excised Node) }\end{array}$ & Stage & $\begin{array}{c}\text { Clinical } \\
\text { Response }\end{array}$ \\
\hline CHL-64 & MCCHL & POS & & 6 & F & Neck & $3 B$ & CR \\
\hline
\end{tabular}

NA $=$ Not available

Disease Subtypes $(n=54)$

- $\mathrm{NSCHL}=$ Nodular Sclerosis Classical Hodgkin Lymphoma $(\mathrm{n}=30 / 55.6 \%)$

- $\mathrm{MCCHL}=$ Mixed Cell Classical Hodgkin Lymphoma $(n=15 / 27.8 \%)$

- $\mathrm{LRCHL}=$ Lymphocyte rich Classical Hodgkin Lymphoma ( $\mathrm{n}=3 / 5.6 \%)$

- CHL-NOS $=$ Classical Hodgkin Lymphoma, Not otherwise specified $(n=6 / 11.1 \%)$

\section{EBV Status}

- Epstein-Barr virus, as assessed by Epstein-Barr virus (EBV)-encoded small RNAs (EBERs).

\section{GeoMx}

- Tumor biopsies selected for Nanostring GeoMx assay.

\section{Stage}

- Ann Arbor staging classification of lymphoma

\section{Clinical Response}

- Best response with first-line treatment / any further event and treatment

- $\mathrm{CR}=$ complete remission or complete metabolic response

- $\mathrm{PD}=$ progesssive disease

Table 2 - Antibodies and secondary reagents used for multiplex immunofluorescence panels 


\begin{tabular}{|l|l|l|l|l|l|l|}
\hline \multicolumn{1}{|c|}{$\begin{array}{c}\text { Primary } \\
\text { Antibody }\end{array}$} & \multicolumn{1}{|c|}{$\begin{array}{c}\text { Primary Antibody } \\
\text { Dilution }\end{array}$} & \multicolumn{1}{|c|}{ Clone } & \multicolumn{1}{|c|}{ Manufacturer } & $\begin{array}{c}\text { Secondary } \\
\text { Antibody } \\
\text { (Ventana) }\end{array}$ & $\begin{array}{c}\text { TSA- } \\
\text { Conjugated } \\
\text { Fluorochrome }\end{array}$ \\
\hline 1 & CD11c & $1: 200$ & 5 D11 & Leica & Omnimap & Opal-520 \\
\hline 2 & CD30 & $1: 25$ & BerH2 & Cell Marque & Ultramap & Opal-540 \\
\hline 3 & CD68P $^{\square}$ & $1: 1500$ & PGM1 & Dako & Omnimap & Opal-570 \\
\hline 4 & IDO1 & $1: 4000$ & V1NC3IDO & Invitrogen & Omnimap & Opal-620 \\
\hline 5 & PD-L1 ${ }^{\square}$ & Pre-dilute & SP263 & Ventana & Optiview & Opal-650 \\
\hline 6 & TIM-3 & $1: 200$ & D5D5R & $\begin{array}{l}\text { Cell Signaling } \\
\text { Technology }\end{array}$ & Omnimap & Opal-690 \\
\hline 7 & CADM1* & $1: 10,000$ & Polyclonal & Sigma-Aldrich & Omnimap & Opal-570 \\
\hline 8 & CD1C* & $1: 100$ & OTI2F4 & Abcam & Omnimap & Opal-620 \\
\hline 9 & LAMP3* & $1: 100$ & 1010E1 & Dendritics & Ultramap & Opal-650 \\
\hline 10 & CD123* & $1: 200$ & BR4MS & Leica & Optiview & Opal-690 \\
\hline
\end{tabular}

TSA = Tyramide signal amplification.

CD11c \& CD30 are used in both multiplexes. Other antibodies are annotated, as below:

口 Multiplex 1 only

* Multiplex 2 only

Table 3 - Antibodies and secondary reagents used for multiplex immunofluorescence panels 


\begin{tabular}{|l|l|l|l|l|l|l|}
\hline & \multicolumn{1}{|c|}{$\begin{array}{c}\text { Primary } \\
\text { Antibody }\end{array}$} & \multicolumn{1}{|c|}{$\begin{array}{c}\text { Primary Antibody } \\
\text { Dilution }\end{array}$} & \multicolumn{1}{|c|}{ Clone } & \multicolumn{1}{|c|}{ Manufacturer } & $\begin{array}{c}\text { Secondary } \\
\text { Antibody } \\
\text { (Ventana) }\end{array}$ & $\begin{array}{c}\text { TSA- } \\
\text { Conjugated } \\
\text { Fluorochrome }\end{array}$ \\
\hline 1 & CD11c & $1: 200$ & 5 D11 & Leica & Omnimap & Opal-520 \\
\hline 2 & CD30 & $1: 25$ & BerH2 & Cell Marque & Ultramap & Opal-540 \\
\hline 3 & CD68 $^{\square}$ & $1: 1500$ & PGM1 & Dako & Omnimap & Opal-570 \\
\hline 4 & IDO1 & $1: 4000$ & V1NC3IDO & Invitrogen & Omnimap & Opal-620 \\
\hline 5 & PD-L1 & Pre-dilute & SP263 & Ventana & Optiview & Opal-650 \\
\hline 6 & TIM-3 & $1: 200$ & D5D5R & $\begin{array}{l}\text { Cell Signaling } \\
\text { Technology }\end{array}$ & Omnimap & Opal-690 \\
\hline 7 & CADM1* & $1: 10,000$ & Polyclonal & Sigma-Aldrich & Omnimap & Opal-570 \\
\hline 8 & CD1C* & $1: 100$ & OTI2F4 & Abcam & Omnimap & Opal-620 \\
\hline 9 & LAMP3* & $1: 100$ & 1010E1 & Dendritics & Ultramap & Opal-650 \\
\hline 10 & CD123* & $1: 200$ & BR4MS & Leica & Optiview & Opal-690 \\
\hline
\end{tabular}

TSA $=$ Tyramide signal amplification.

CD11c \& CD30 are used in both multiplexes. Other antibodies are annotated, as below:

口 Multiplex 1 only

* Multiplex 2 only

Table 4. Outline of the merged single cell RNA sequencing dataset

\begin{tabular}{|l|l|c|c|l|}
\hline & Histology & Number of cases & Cell Number & Data source \\
\hline \multirow{3}{*}{ Hodgkin Lymphoma } & NSCHL & 13 & 59,885 & This study \& Aoki et al \\
\cline { 2 - 5 } & MCCHL & 9 & 38,046 & Aoki et al \\
\cline { 2 - 5 } & LRCHL & 1 & 4,683 & Aoki et al \\
\cline { 2 - 5 } & NLPHL & 1 & 21,862 & This study \\
\hline \multirow{2}{*}{ Non-Lymphoma } & Healthy & 8 & 79,823 & This study \\
\cline { 2 - 5 } & Reactive & 5 & 23,187 & Aoki et al \\
\hline
\end{tabular}


Histological Subtypes of Hodgkin Lymphoma

- $\mathrm{NSCHL}=$ Nodular Sclerosis Classical Hodgkin Lymphoma

- $\mathrm{MCCHL}=$ Mixed Cell Classical Hodgkin Lymphoma

- $\mathrm{LRCHL}=$ Lymphocyte rich Classical Hodgkin Lymphoma

- $\mathrm{NLPHL}=$ Nodular lymphocyte predominant Hodgkin Lymphoma

\section{Figures}


Figure 1

a

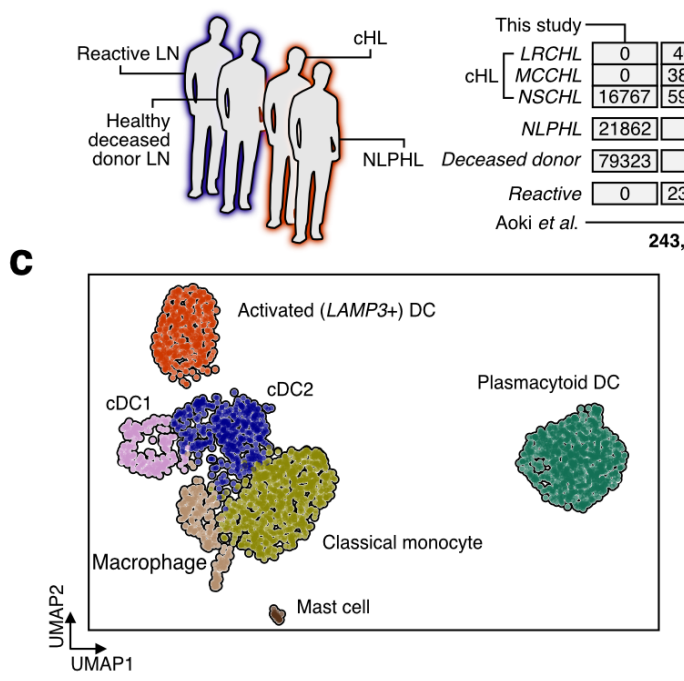

b

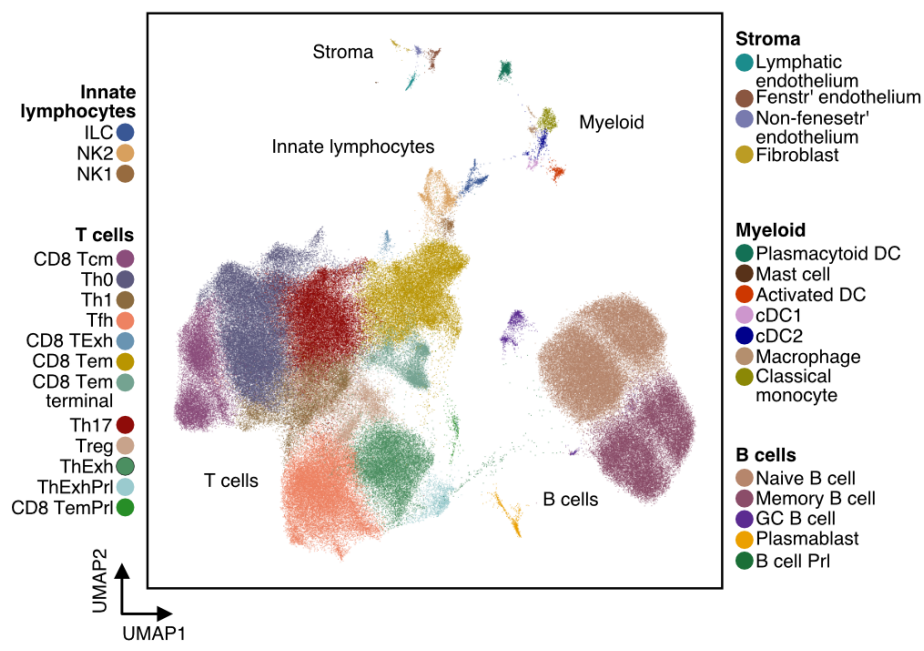

e

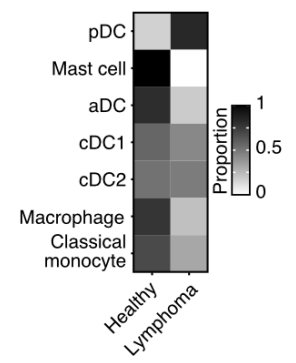

h

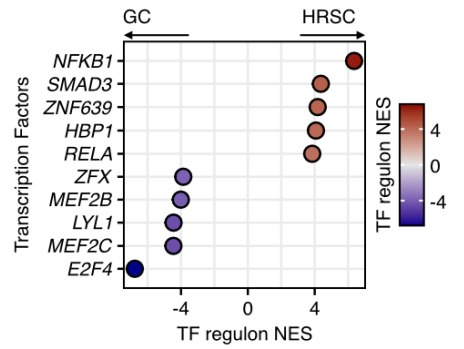

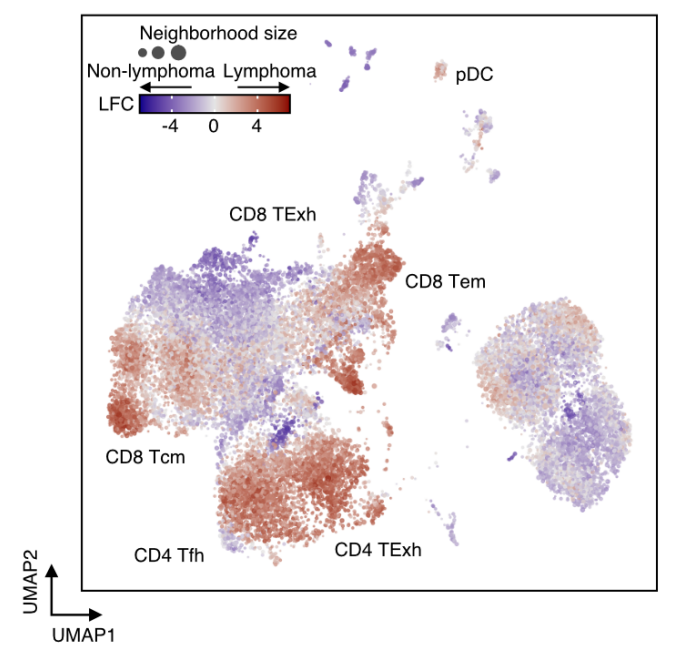

9

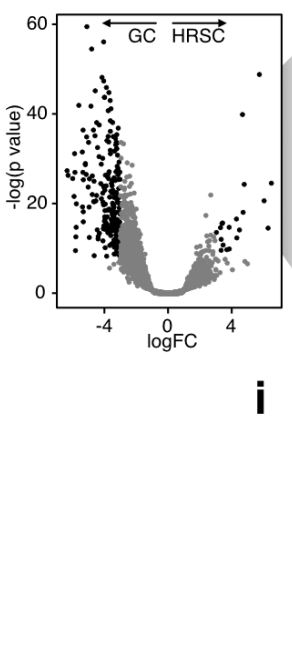

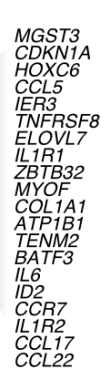

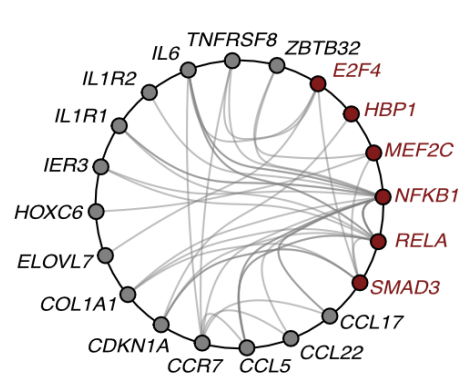

Figure 1

Study design and transcriptional profiling of immune and neoplastic cells in the Classic Hodgkin lymphoma microenvironment. a. Outline of the scRNAseq dataset. Two datasets (Aoki et al. and "this study"/WSI dataset) were merged, and contain data from lymph nodes unaffected by lymphoma (reactive lymph nodes and non-diseased lymph nodes from deceased donors) highlighted in blue, and lymphoma-infiltrated lymph nodes highlighted in red. The numbers of cells are given in dark gray, and the 
number of donors in light gray. b. UMAP plot of 243,753 cells from an integrated scRNAseq dataset, colored by cell-type and organized by compartment (T-cells, B cells, innate lymphocytes, myeloid, and stroma). c. UMAP plot of 2727 myeloid cells colored by cell-type. $\mathbf{d}$. Heatmap showing mean normalized expression levels (color) and fraction of cells expressing (dot size) markers of myeloid cell subsets. e. Heatmap showing the proportion of each cell-type derived from healthy and lymphoma samples. $f$. UMAP plot of 22,573 graph neighborhoods, colored by the differential abundance (log fold change) in lymphoma affected or non lymphoma affected (deceased-donor lymph nodes or reactive lymph node) samples. Dot size is proportional to neighborhood size (median neighborhood size $=50$ cells). g. Volcano plot showing differentially expressed genes between microdissected GC and HRSC. Significant genes for HRSC are indicated $(\mid \mathrm{LFCl}>3$, adjusted $p$ value $<0.01)$. $\mathbf{h}$. Heatmap showing transcription factor $(T F)$ regulon normalized enrichment scores for GC vs HRSC calculated with DoRothEA. i. Graph indicating predicted interactions between genes (red, transcription factors; gray, other genes) differentially expressed in HRSC. Edges drawn where an interaction is documented by OmniPath. 
Figure 2

a

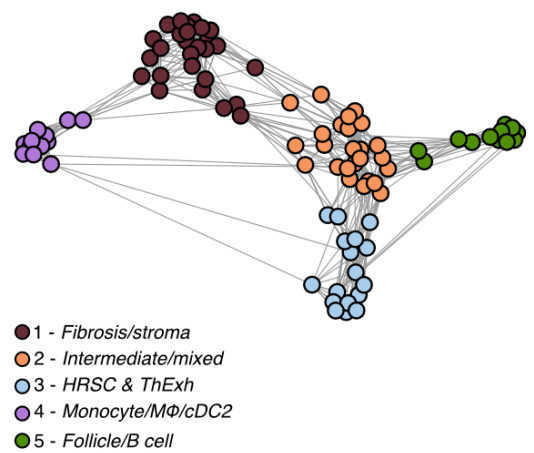

b

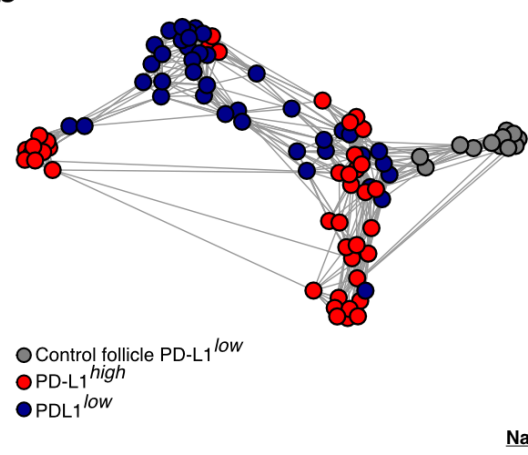

C

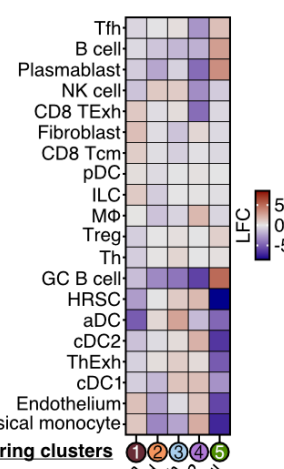

d

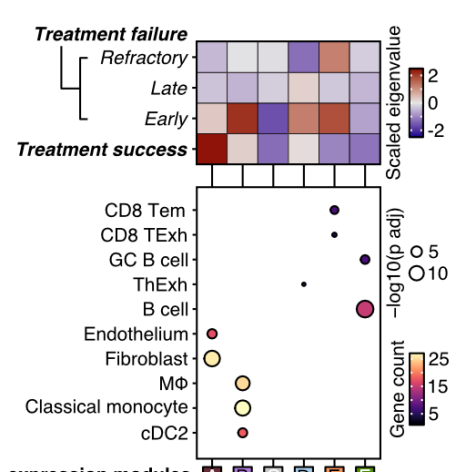

Co-expression modules 亩直直直南

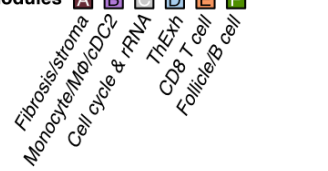

f

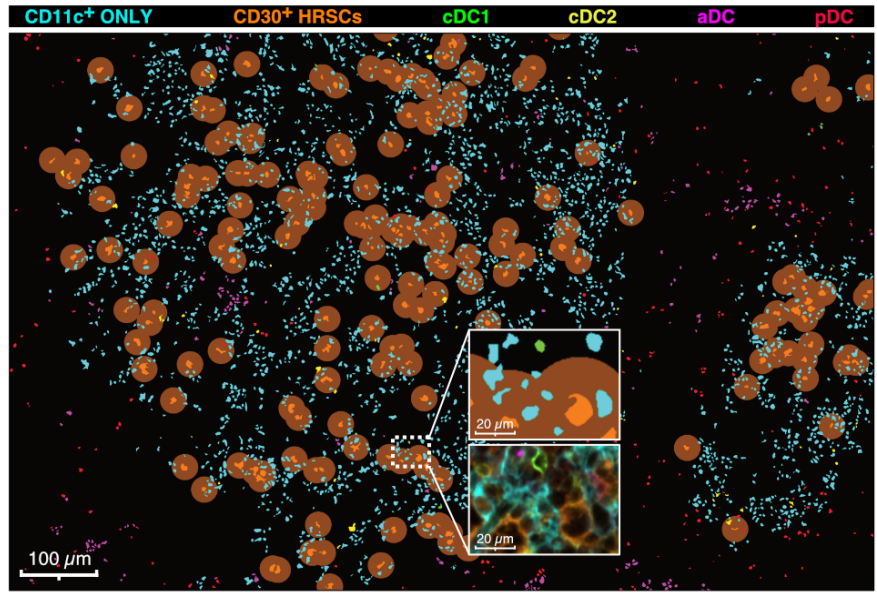

9

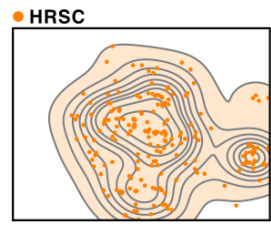

CD11C+ ONLY
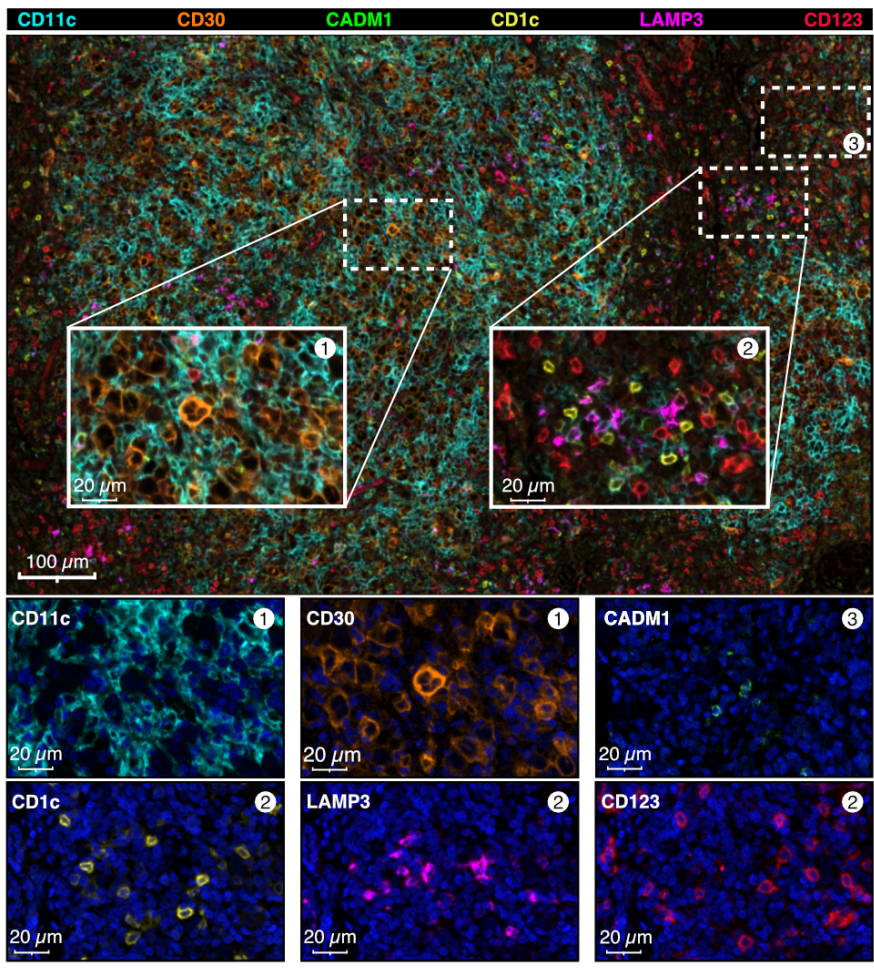

h
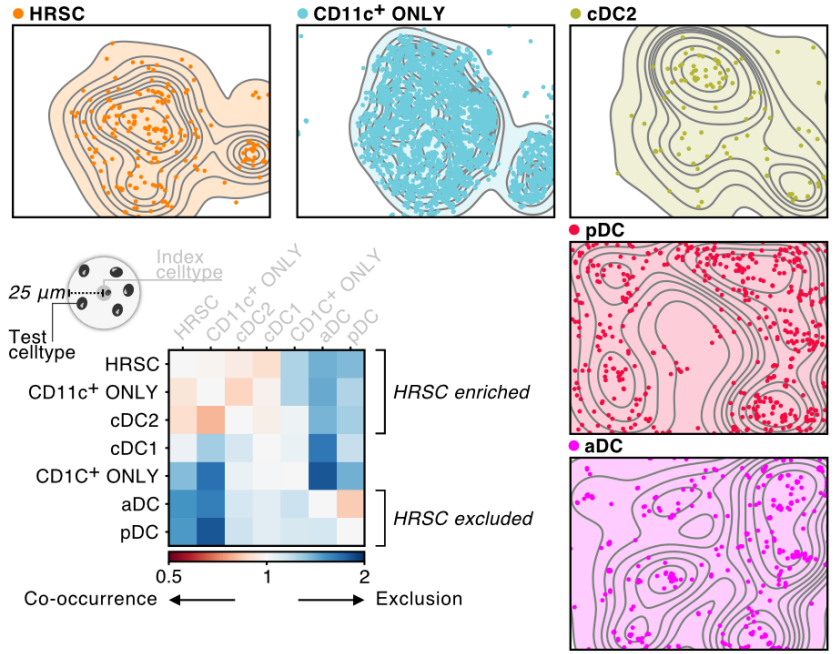

Figure 2

Heterogeneous immune cell networks occupy distinct tissue niches with divergent mononuclear phagocyte enrichment patterns and prognostic significance. a. Shared nearest-neighbor graph embedding of transcriptional profiles of nanostring ROI from reactive and $\mathrm{cHL}$ lymph nodes. Color indicates clusters identified using Leiden clustering with resolution $=1$. b. Shared nearest-neighbor graph embedding of transcriptional profiles of nanostring ROI colored by PD-L1 expression status. C. 
Differential abundance estimates of cell-types deconvolved from nanostring ROI between clusters shown in (a), LFC estimates represent differential abundance of cell-types in each cluster vs all other clusters. (MФ, Macrophage) d. Upper panel: Heatmap showing WGCNA module coexpression patterns for 6 modules (modules A-F) across $130 \mathrm{cHL}$ gene expression profiles (microarray) split by treatment outcome. Lower panel: Heatmap showing results for an hypergeometric enrichment test between module gene membership and cell type markers from the scRNAseq dataset for WGCNA modules (modules A-F). (MФ, Macrophage). e. Representative micrographs from multiplexed IF imaging of $\mathrm{cHL}$ samples. One of 3 test regions from tumor CHL-27 is shown, cropped to $1662 \mu \mathrm{m}$ by $1076 \mu \mathrm{m} ; \mathrm{CD} 11 \mathrm{c}$ (cyan), CD30 (orange), CADM1 (green), CD1c (yellow), LAMP3 (magenta), and CD123 (red) fluorescent signals are represented by unique pseudocolors. To improve clarity DAPI is not shown. Selected areas are highlighted, each at 100x magnification $(160 \mu \mathrm{m} \times 108 \mu \mathrm{m})$; CD30+ HRSC-dense area (region 1, inset left), internodular area with no CD30+ HRSC (region 2, inset right), and area with CADM1+ CDC1 (region 3, inset top right). Individual pseudocolors are shown below the main image (second \& third lines), with DAPI (blue) to identify cell nuclei. Each individual image refers to a multiplexed area above (inset), indicated by the corresponding number (top right). f. HRSC and DC map, corresponding to the same region from (e). Phenotyped cells are identified by colored nuclei, with each cell-type represented by a different color; 'CD11c+ ONLY' (CD11c+ CD1c-, cyan), CD30+ HRSC (light orange), CDC1 (green), CDC2 (yellow), LAMP3+ aDC (magenta), and pDC (red) are shown. Cells with no assigned phenotype ('null') and 'CD1c+ ONLY' cells are excluded from this visualization. The 'HRSC neighborhood' is shown as circles surrounding each CD30+ HRSC (dark orange). A selected area is highlighted, at 200x magnification ( $80 \mu \mathrm{m} \times 54 \mu \mathrm{m}$; inset, center); $\mathbf{g}$. Isobar plots show the location and density of each cell phenotype of interest, for the corresponding tumor region from (e) and (f) (note the uncropped $2008 \mu \mathrm{m}$ by $1502 \mu \mathrm{m}$ region is shown here). HRSCs (orange dots, left) and DC subsets (colors corresponding to each phenotype, right) are shown. h. Summarized interaction plot across all study tumors in aggregate, displaying the ratio of the nearest neighbor distance between phenotype pairs (index cell-type (gray) to test cell-type (black)) compared to the expected baseline distance. Red indicates cell-type co-occurrence, blue indicates celltype exclusion. 

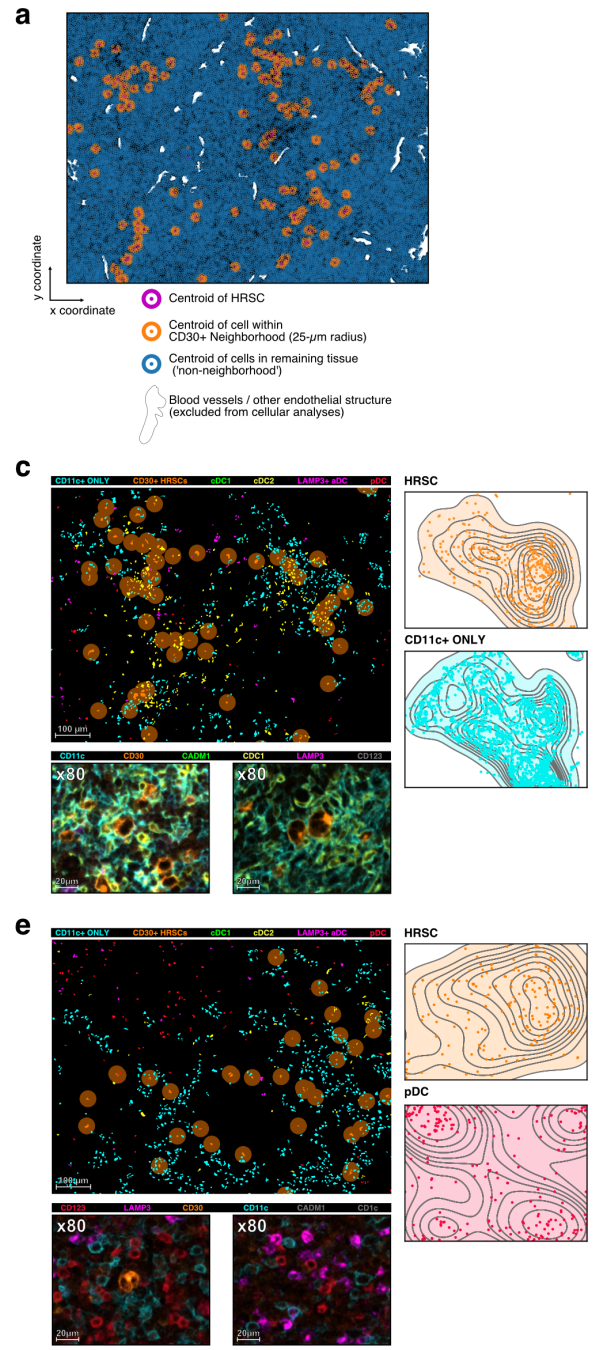

g
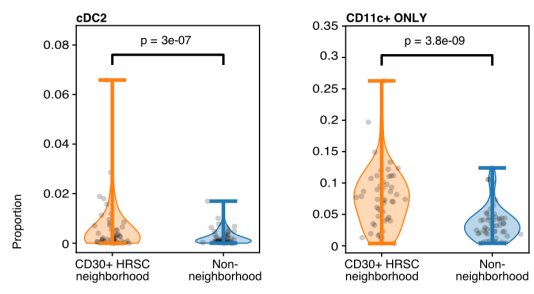

h
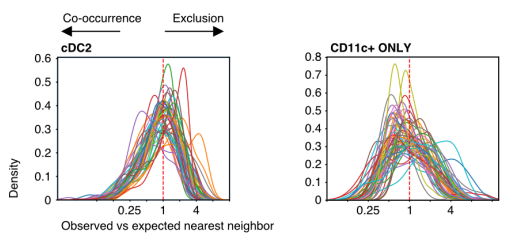
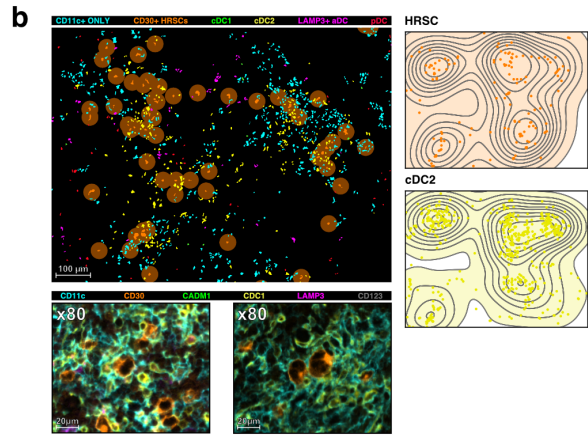

d
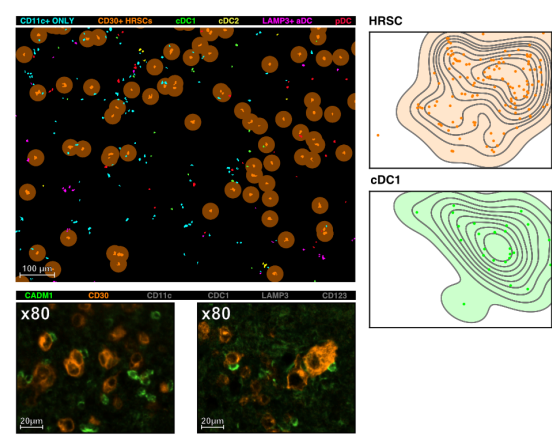

f
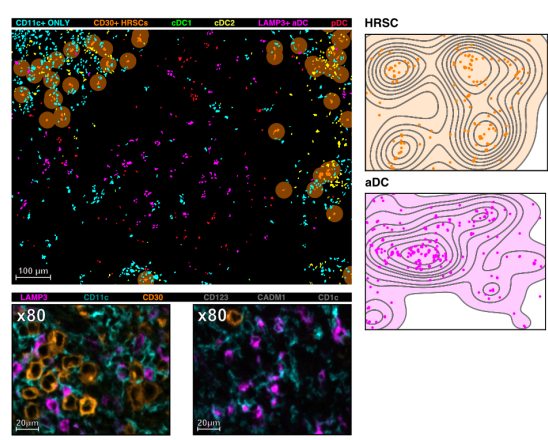
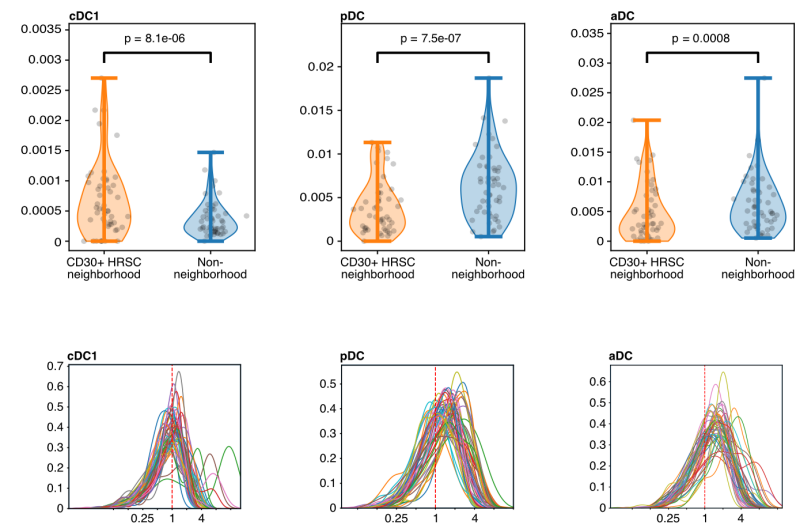

Figure 3

Plasmacytoid DC and activated DC are excluded from the Hodgkin Reed-Sternberg cell microenvironment, but immunosurveillant $\mathrm{CDC1}$ and $\mathrm{CDC} 2$ are enriched. a. Representative region from $\mathrm{CHL}-13$, which measures $2008 \mu \mathrm{m}$ (x-axis) by $1502 \mu \mathrm{m}$ (y-axis). The centroid of each cell is identified with a colored circle. The CD30+ HRSC are indicated by a magenta circle. The cell nuclei which lie within a $10-25-\mu \mathrm{m}$ radius from each HRSC centroid ('HRSC neighborhood') are indicated by orange circles and those nuclei 
beyond the $25-\mu \mathrm{m}$ areas ('non-neighborhood') are indicated by blue circles. Blood vessels / other endothelial structures are indicated (white) and all cells encompassed within these areas are excluded from analysis. b, c, d. Spatial distribution of dendritic cell subtypes that co-occur with HRSC. For each phenotype a representative tumor region is identified and shown in all plots: $\mathbf{b} . \mathrm{cDC} 2(\mathrm{CD} 11 \mathrm{c}+\mathrm{CD} 1 \mathrm{c}+)=$ represented by CHL-13. c. CD11 + ONLY cells $=$ CHL-43. d. $C D C 1=C H L-31$. e, f. Spatial distribution of dendritic cell subtypes that are 'excluded' from HRSC. For each phenotype a representative tumor region is identified and shown in all plots: e. $\mathrm{pDC}=\mathrm{CHL}-11$. $\mathbf{f}$. aDC / LAMP3 $+=\mathrm{CHL}-13$. A cropped, illustrative area from each tumor region (measuring $1004 \mu \mathrm{m}$ by $751 \mu \mathrm{m}$, left) is shown as a cell map; phenotyped cells are identified by colored nuclei, with each cell-type represented by a different color. 'CD11c+ ONLY' (cyan), CD30+ HRSC (light orange), cDC1 (green), cDC2 (yellow), LAMP3+ aDC (magenta), and pDC (red) are shown. Cells with no assigned phenotype ('null') and 'CD1C+ONLY' cells are excluded from this visualization. The 'HRSC neighborhood' is shown as circles surrounding each CD30+ HRSC (dark orange), each circle extending to a radius of $25 \mu \mathrm{m}$ around each HRSC. Topological maps of the corresponding full region of interest (2008 $\mu \mathrm{m}$ by $1502 \mu \mathrm{m})$ of HRSC (orange, upper right), immune cells of interest (colored according to cell phenotype, lower right). Representative immunofluorescence images (80x magnification), showing cells of interest (second line). CD11c (cyan), CD30 (orange), CADM1 (green), CD1c (yellow), LAMP3 (magenta), and CD123 (red) fluorescent signals are represented by unique pseudocolors. To improve visualization, certain signals may not be shown in each particular image (these excluded colors are shown in gray in each respective key). g. Violin plots comparing the observed cell proportions for each tumor sample for the CD30+ HRSC Neighborhood (orange) and across the entire examined tissue area ('Non-neighborhood'; blue), for each DC subtype. Statistical significance: QQ plots showed that the differences in cell proportions between the CD30 neighborhood and the entire sample were not normally distributed, so a Wilcoxon signed-rank test was used to assess statistical significance for each phenotype. $\mathbf{h}$. Histograms showing the distance from each HRSC to its nearest-neighbor of an immune-phenotype. Distance is shown as a ratio to the expected distance if the immune cell were distributed randomly within the tissue section, with values less than 1 (left of dotted line) indicating that cells co-occur with HRSC and values greater than 1 (right of dotted line) indicating that cells are excluded from HRSC. 
Figure 4

a

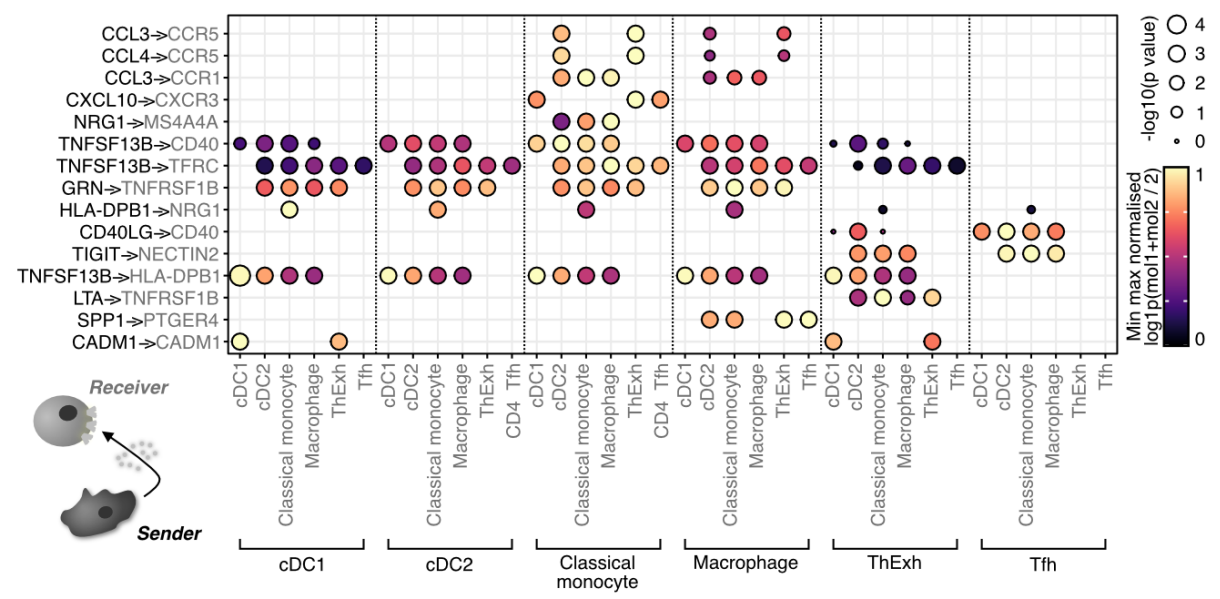

b

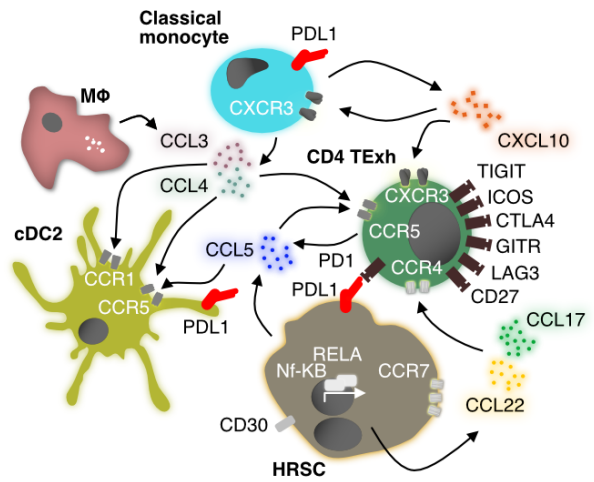

Figure 4

Reciprocal mononuclear phagocyte chemokine signaling shapes the immunosuppressive microenvironment in cHL.a. Heatmap of reciprocal ligand-receptor interactions between myeloid subsets and T-cells implicated in the cHL TME. Point size indicates permutation $\mathrm{p}$ value (CellPhoneDB). Color indicates the min-max normalized aggregate mean expression level of ligand and receptor. $\mathbf{b}$. Model for intercellular interactions between MNP, dysfunctional T-cells, and HRSC in the CHL TME. HRSC produce 
CCL22, CCL17, and CCL5 downstream of NF-KB activity, orchestrating T-cell recruitment via indicated ligand-receptor interactions. CCL3, CCL4, and CXCL10 produced by monocytes and macrophages directs positioning of dysfunctional T-cells and CDC2. Widespread inhibitory molecule expression is seen on HRSC, MNP, and T-cells.

Figure 5
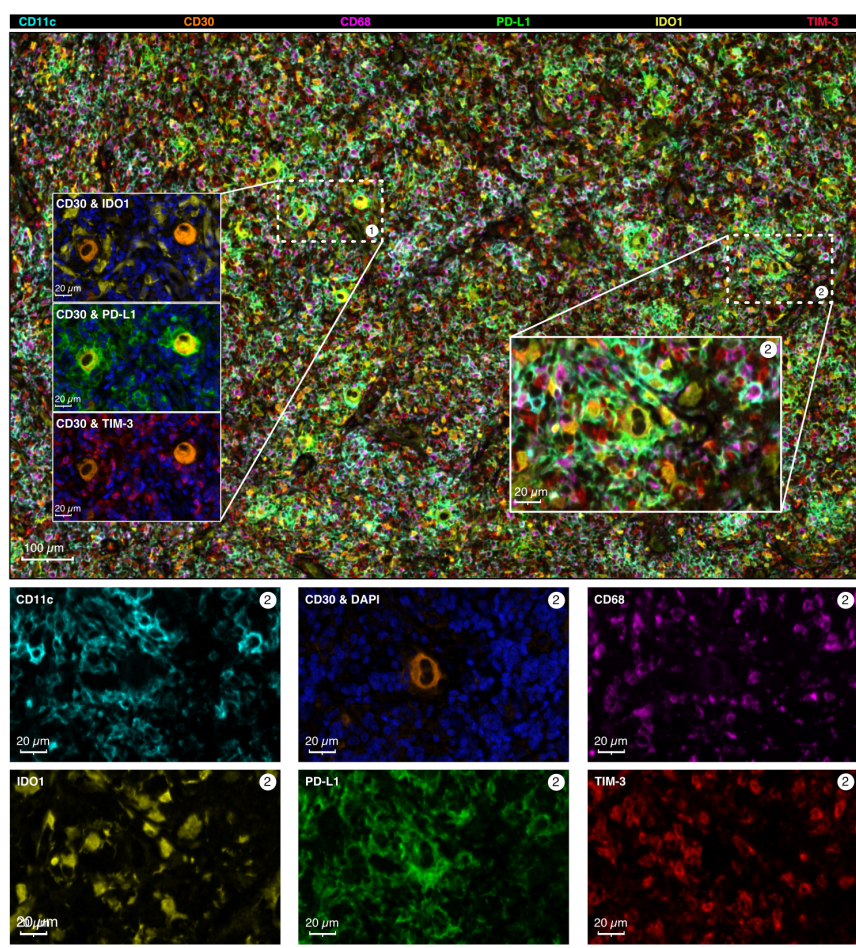

d

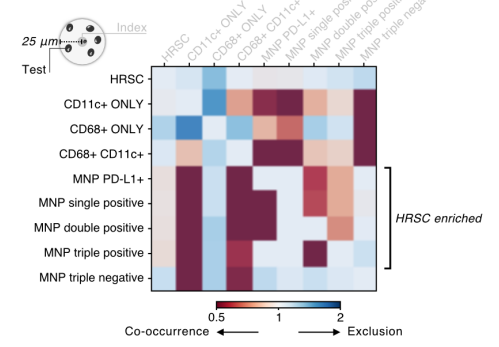

b
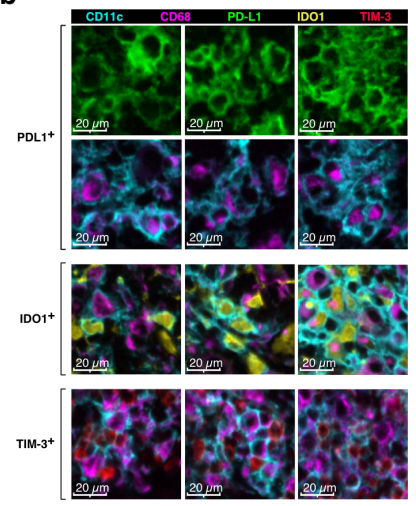

c
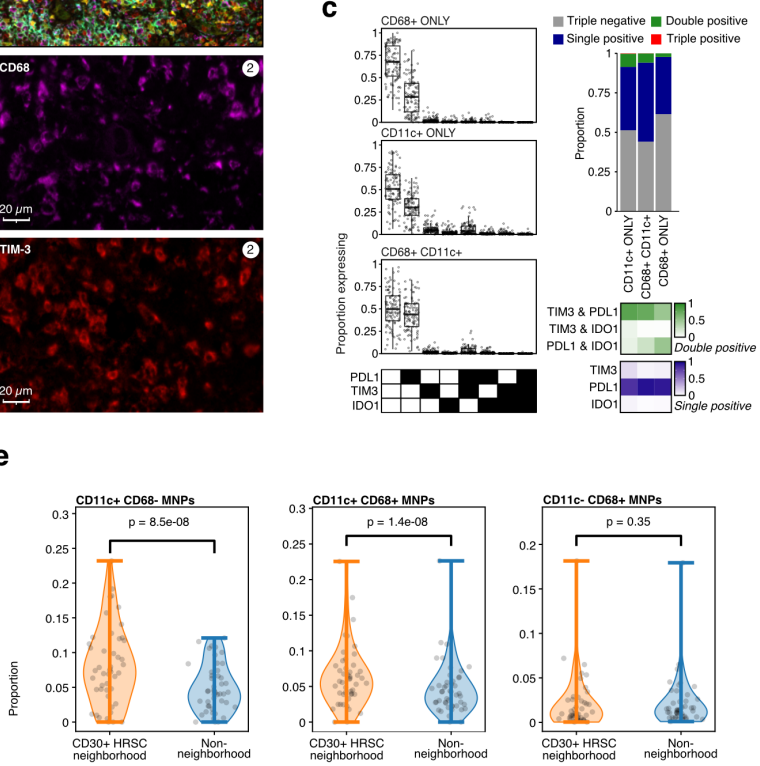

Figure 5 
Mononuclear phagocytes expressing one or more immunosuppressive molecules are enriched around Hodgkin Reed-Sternberg cells in cHL. a. Representative micrographs from multiplexed IF imaging of $\mathrm{cHL}$ samples, showing expression of inhibitory molecules (ID01, PD-L1, \& TIM-3), cell-type defining markers for MNPs (CD11c \& CD68), and HRSC (CD30). One of 3 test regions from CHL-7 is shown $(2008 \mu \mathrm{m} \mathrm{x}$ $1502 \mu \mathrm{m})$, with CD11c (cyan), CD30 (orange), CD68 (magenta), PD-L1 (green), ID01 (yellow), and TIM-3 (red) fluorescent signal represented by unique pseudocolors (to improve clarity DAPI is not shown). Inset 1 (100x magnification [160 $\mu \mathrm{m} \times 108 \mu \mathrm{m}]$; left) shows CD30 and ID01 (top), CD30 and PD-L1 (middle), and CD30 and TIM-3 (bottom), with DAPI (blue) to identify cell nuclei. Inset 2 (100x magnification [160 $\mu \mathrm{m} \times 108 \mu \mathrm{m}]$, right) shows representative CD30+ HRSC, surrounded by mononuclear phagocytes in closer detail. Individual pseudocolors from area 2, corresponding to each antibody, are shown below the main image (second \& third lines). b. Expression of PD-L1, ID01, and TIM-3 by MNPs. Representative images at 200x magnification (54 $\mu \mathrm{m} \times 54 \mu \mathrm{m})$ show PD-L1 expression (green, top line) and corresponding CD11c (cyan) and CD68 (magenta, second line) for the same areas. Images are separated to improve clarity, as co-localized. ID01 expression (yellow, cytoplasmic localization) with CD11c and CD68 (third line). TIM-3 expression (red, cytoplasmic localization) with CD11c and CD68 (bottom line). c. Proportion of mononuclear phagocytes expressing immuno-regulatory molecules. Left panel: lower key represents the combinations of inhibitory molecules (black, expressed; white, unexpressed) corresponding to the proportions shown in boxplots, individual points correspond to $\mathrm{CHL}$ cases. Right upper panel: proportions of each cell-type expressing, none, one, two or three inhibitory molecules in aggregate across all images. Right lower panel: Proportion of single or double positive cells expressing each indicated combination of inhibitory molecules. d. Summarized interaction plot across all study tumors in aggregate, displaying the ratio of the nearest neighbor distance between phenotype pairs (index phenotype (gray) to test phenotype (black)) compared to the expected baseline distance. Red indicates cell-type co-occurrence, blue indicates cell-type exclusion. e. Violin plots comparing the observed cell proportions for each tumor sample for the CD30+ Neighborhood (orange) and across the entire examined tissue area ('Non-neighborhood'; blue), for each MNP subtype. Wilcoxon signed-rank test used to assess statistical significance.

\section{Supplementary Files}

This is a list of supplementary files associated with this preprint. Click to download.

- SuppfigS1.tiff

- SuppfigS2.tiff

- SuppfigS3.tiff

- SuppfigS4.tiff

- SuppfigS5.tiff

- SuppfigS6.tiff

- visualabstract.tiff 